\title{
Hall Currents and Heat Transfer Effects on Peristaltic Transport in a Vertical Asymmetric Channel through a Porous Medium
}

\author{
E. Abo-Eldahab, ${ }^{1}$ E. Barakat, ${ }^{1}$ and Kh. Nowar ${ }^{2}$ \\ ${ }^{1}$ Department of Mathematics, Faculty of Science, Helwan University, Helwan 11795, Egypt \\ ${ }^{2}$ Department of Mathematics, Faculty of Science, King Saud University, P.O. Box 2455, \\ Riyadh 11451, Saudi Arabia \\ Correspondence should be addressed to Kh. Nowar, khalidnowar@yahoo.com
}

Received 28 December 2011; Accepted 27 February 2012

Academic Editor: Hung Nguyen-Xuan

Copyright (C) 2012 E. Abo-Eldahab et al. This is an open access article distributed under the Creative Commons Attribution License, which permits unrestricted use, distribution, and reproduction in any medium, provided the original work is properly cited.

\begin{abstract}
The influences of Hall currents and heat transfer on peristaltic transport of a Newtonian fluid in a vertical asymmetric channel through a porous medium are investigated theoretically and graphically under assumptions of low Reynolds number and long wavelength. The flow is investigated in a wave frame of reference moving with the velocity of the wave. Analytical solutions have been obtained for temperature, axial velocity, stream function, pressure gradient, and shear stresses. The trapping phenomenon is discussed. Graphical results are sketched for various embedded parameters and interpreted.
\end{abstract}

\section{Introduction}

Peristalsis is a phenomenon found in several physiological and industrial processes. The word peristalsis stems from the Greek word "Peristalikos" which means clasping and compressing.

Peristalsis is an important mechanism for mixing and transporting fluids that occurs when a progressive wave of area contraction or expansion propagates along the wall of the tube. Peristaltic flows occur widely in chemical processes such as in distillation towers and fixed-bed reactors, urine transport from kidney to bladder through the ureter, transport of lymph in the lymphatic vessels, swallowing food through the esophagus, the movement of chyme in the gastrointestinal tract, ovum movement in the fallopian tube, transportation of spermatozoa in the ductus efferentes of the male reproductive tracts, in the vasomotion of small blood vessels, transport of corrosive fluids, in sanitary fluid transport, and blood pumps in heart lung machine. In addition, peristaltic pumping occurs in many practical applications involving biomechanical systems. Several attempts had been made to know 
and understand peristaltic action in different situations. Since the first attempt of Latham [1] some interesting investigations in this direction have been given in [2-17]. Flow through a porous medium attracted the attention of many researchers in the last few decades because of its very important practical applications. It occurs in filtration of fluids and seepage of water in river beds, sandstone, limestone, bile duct, wood, the human lung, gall bladder with stones, and in small blood vessels. El Shehawey and Husseny [18] formulated a mathematical model for the peristaltic transport of a viscous incompressible fluid through a porous medium bounded by two porous plates. El Shehawey et al. [19] studied the peristaltic transport in an asymmetric channel through a porous medium. Vajravelu et al. [20] have examined flow through vertical porous tube with peristalsis and heat transfer. Srinvas and Kothandapani [21] have studied the influence of heat and mass transfer on MHD peristaltic flow through a porous space with compliant walls. Srinvas and Gayathri [22] have discussed the peristaltic transport of Newtonian fluid in a vertical asymmetric channel with heat transfer and porous medium. The Hall effect is important when the Hall parameter which is the ratio between the electron-cyclotron frequency and the electron-atom-collision frequency is high; this can occur if the collision frequency is low or when the magnetic field is high. This is a current trend in magnetohydrodynamics because of its important influence of the electromagnetic force. Hence, it is important to study Hall effects and heat transfer effects on the flow to be able to determine the efficiency of some devices such as power generators and heat exchangers. Attia [23] had examined unsteady Hartmann flow with heat transfer of a viscoelastic fluid taking the Hall effect into account. Asghar et al. [24] studied the effects of Hall current and heat transfer on flow due to a pull of eccentric rotating disk. Hayat et al. [25] studied the Hall effects on peristaltic flow of a Maxwell fluid in a porous medium. Abo-Eldahab et al. [26, 27] investigated the effects of Hall and ion-slip currents on magnetohydrodynamic peristaltic transport and couple stress fluid. For the benefit of the readers in this direction you can take these additional papers [28-35] into account. This paper may be considered as an extension of the paper [22] by Srinvas and Gayathri, if we neglected the effect of Hall current.

The aim of this paper is to investigate the effects of Hall current and heat transfer on peristaltic transport of a Newtonian fluid in a vertical asymmetric channel through a porous medium. We introduce the governing equations and boundary conditions in Section 2. Section 3 represents the volume flow rate. The exact solution of the problem is derived in Section 4 . Section 5 deals with numerical results and discussion. The trapping phenomenon is discussed in Section 6. The conclusions are summarized in Section 7.

\section{Problem Formulation}

Consider the peristaltic flow of an incompressible viscous fluid in a two-dimensional vertical infinite asymmetric channel of width $d_{1}+d_{2}$ through a porous medium. Asymmetry in the channel is produced by choosing the peristaltic wave trains propagating with constant speed $c$ along the walls ( $H_{1}$ is the right hand side wall and $H_{2}$ is the left hand side wall) to have different amplitudes and phases as shown in Figure 1.

The shapes of the channel walls are represented as

$$
\begin{gathered}
Y=H_{1}(X, t)=d_{1}+a_{1} \cos \left(\frac{2 \pi}{\lambda}[X-c t]\right), \text { right wall, } \\
Y=H_{2}(X, t)=-d_{2}-b_{1} \cos \left(\frac{2 \pi}{\lambda}[X-c t]+\phi\right), \text { left wall, }
\end{gathered}
$$




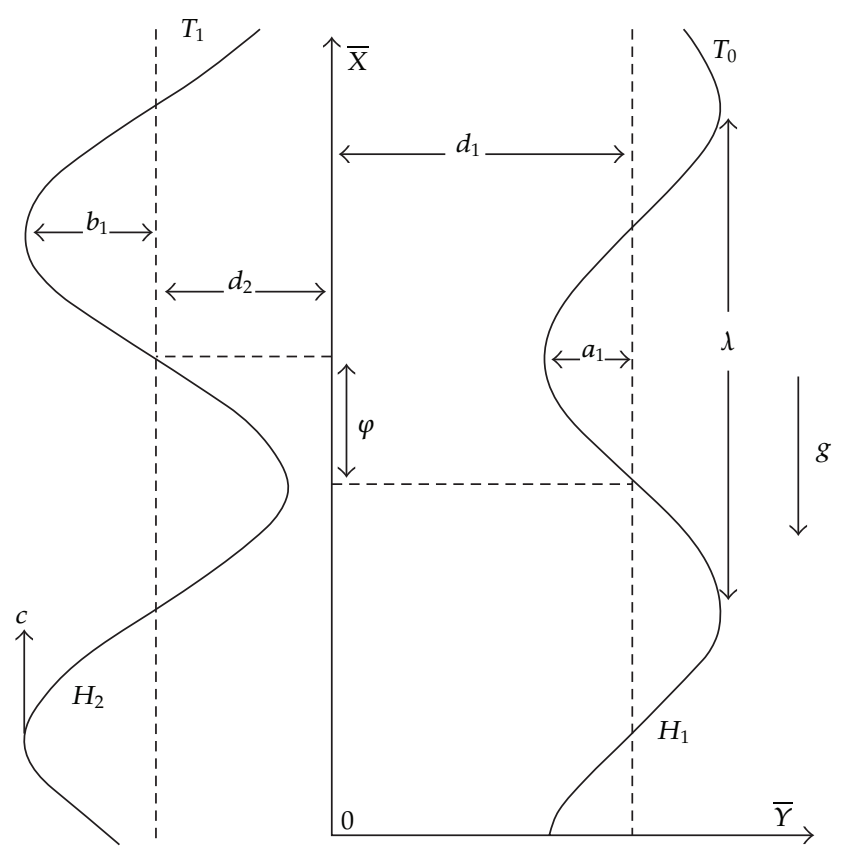

Figure 1: The geometry of the problem.

where $a_{1}, b_{1}$ are the amplitudes of the right and left waves, $l$ is the wave length, the phase difference $\phi$ varies in the range $0 \leq \phi \leq \pi, \phi=0$ corresponds to symmetric channel with waves out of phase, and $\phi=\pi$ describes the case where waves are in phase. Further, $d_{1}, d_{2}$, $a_{1}, b_{1}$, and $\phi$ satisfy the following inequality:

$$
a_{1}^{2}+b_{1}^{2}+2 a_{1} b_{1} \cos \phi \leq\left(d_{1}+d_{2}\right)^{2},
$$

so that the walls will not intersect with each other. The heat transfer in the channel is taken into account by maintaining the right and left walls at temperatures $T_{0}$ and $T_{1}$, respectively. A uniform magnetic field with magnetic flux density vector $\mathbf{B}=\left(0,0, B_{0}\right)$ is applied, and the induced magnetic field is neglected by taking a very small magnetic Reynolds number. The fundamental equations governing this model together with the generalized Ohm's law taking the effects of Hall currents and Maxwell's equations into account are

$$
\begin{gathered}
\nabla \cdot \mathbf{V}=0, \\
-\nabla P+\mu \nabla^{2} \mathbf{V}+\rho g \alpha\left(T-T_{0}\right)-\frac{\mu}{k_{1}} \mathbf{V}+\mathbf{J} \wedge \mathbf{B}=\rho \frac{d \mathbf{V}}{d t}, \\
\mathbf{J}=\sigma\left[\mathbf{V} \wedge \mathbf{B}-\frac{1}{e n_{e}} \mathbf{J} \wedge \mathbf{B}\right], \\
\rho \zeta \frac{d \mathbf{T}}{d t}=\kappa \nabla^{2} \mathbf{T}+Q_{0},
\end{gathered}
$$

where $\mathbf{V}$ is the velocity vector, $P$ is the pressure, $\mu$ is the dynamic viscosity, $\nabla^{2}$ is the Laplacian operator, $\rho$ is the density of the fluid, $d / d t$ is the material derivative, $t$ is the time, $\mathbf{J}$ is 
the current density, $\mathbf{B}$ is the total magnetic field, $\sigma$ is the electric conductivity, $e$ is the electric charge, $n_{e}$ is the number density of electrons, $\zeta$ is the specific heat at constant pressure, $\alpha$ is the coefficient of linear thermal expansion of the fluid, $\kappa$ is the thermal conductivity of the fluid, $Q_{0}$ is the constant heat addition/absorption, and $T$ is the temperature of the fluid. The equations governing the two-dimensional motion of this model are

$$
\begin{aligned}
\frac{\partial U}{\partial X}+\frac{\partial V}{\partial Y}= & 0 \\
\rho\left(\frac{\partial U}{\partial t}+U \frac{\partial U}{\partial X}+V \frac{\partial U}{\partial Y}\right)= & -\frac{\partial P}{\partial X}+\mu\left(\frac{\partial^{2} U}{\partial X^{2}}+\frac{\partial^{2} U}{\partial Y^{2}}\right)-\frac{\mu}{k_{1}} U+\rho g \alpha\left(T-T_{0}\right) \\
& +\frac{\sigma B_{0}^{2}}{1+m^{2}}(m V-U) \\
\rho\left(\frac{\partial V}{\partial t}+U \frac{\partial V}{\partial X}+V \frac{\partial V}{\partial Y}\right)= & -\frac{\partial P}{\partial Y}+\mu\left(\frac{\partial^{2} V}{\partial X^{2}}+\frac{\partial^{2} V}{\partial Y^{2}}\right)-\frac{\mu}{k_{1}} V-\frac{\sigma B_{0}^{2}}{1+m^{2}}(m U+V), \\
\rho \zeta\left(\frac{\partial T}{\partial t}+U \frac{\partial T}{\partial X}+V \frac{\partial T}{\partial Y}\right)= & \kappa\left(\frac{\partial^{2} T}{\partial X^{2}}+\frac{\partial^{2} T}{\partial Y^{2}}\right)+Q_{0}
\end{aligned}
$$

where $U$ and $V$ are the velocity components in the laboratory frame $(X, Y)$ and

$$
m=\frac{\sigma B_{0}}{e n_{e}} \text { is the Hall parameter. }
$$

Following Shapiro et al. [3] we introduce a wave frame of reference $(x, y)$ moving with velocity $c$ in which the motion becomes independent of time when the channel is an integral multiple of the wavelength and the pressure difference at the ends of the channel is a constant. The transformation from the laboratory frame of reference $(X, Y)$ to the wave frame of reference $(x, y)$ is given by

$$
x=X-c t, \quad y=Y, \quad u=U-c, \quad v=V, \quad p(x)=P(x, t),
$$

where $(u, v), p$ and $(U, V), P$ are the velocity components and pressure in the wave and laboratory frames of reference, respectively.

We introduce the following nondimensional variables:

$$
\begin{aligned}
x^{*}=\frac{x}{\lambda^{\prime}}, \quad y^{*}=\frac{y}{d_{1}}, \quad u^{*}=\frac{u}{c}, \quad v^{*} & =\frac{v}{c \delta}, \quad \delta=\frac{d_{1}}{\lambda}, \quad p^{*}=\frac{p d_{1}^{2}}{c \lambda \mu}, \quad t^{*}=\frac{c t}{\lambda}, \\
h_{1}=\frac{H_{1}}{d_{1}}, \quad h_{2}=\frac{H_{2}}{d_{1}}, \quad d=\frac{d_{2}}{d_{1}}, \quad & a=\frac{a_{1}}{d_{1}}, \quad b=\frac{b_{1}}{d_{1}}, \quad R=\frac{\rho d_{1} c}{\mu}, \quad k=\frac{k_{1}}{d_{1}^{2}}, \\
\theta=\frac{T-T_{0}}{T_{1}-T_{0}}, \quad \operatorname{Pr}=\frac{\mu \zeta}{\kappa}, \quad \beta & =\frac{Q_{0} d_{1}^{2}}{\kappa\left(T_{1}-T_{0}\right)}, \quad G r=\frac{\alpha g\left(T_{1}-T_{0}\right) d_{1}^{3}}{v^{2}}, \\
M & =\frac{\sigma B_{0}^{2} d_{1}^{2}}{\mu},
\end{aligned}
$$


where $R$ is the Reynolds number, $\delta$ is the dimensionless wave number, $k$ is the porosity parameter, Pr is the Prandtl number, $\mathrm{Gr}$ is the Grashof number, $v$ is the kinematic viscosity of the fluid, $M$ is the magnetic parameter, and $\beta$ is the nondimensional heat source/sink parameter. Using (2.6) and (2.7) in (2.4), we can get the following nondimensional form in a wave frame of reference after dropping the stars:

$$
\begin{gathered}
\frac{\partial u}{\partial x}+\frac{\partial v}{\partial y}=0 \\
\delta R\left(u \frac{\partial u}{\partial x}+v \frac{\partial u}{\partial y}\right)=-\frac{\partial p}{\partial x}+\delta^{2} \frac{\partial^{2} u}{\partial x^{2}}+\frac{\partial^{2} u}{\partial y^{2}}-\frac{(u+1)}{k}+\frac{G r \theta}{R}+\delta \frac{m M v}{\left(m^{2}+1\right)}-\frac{M(u+1)}{m^{2}+1} \\
\delta^{3} R\left(u \frac{\partial v}{\partial x}+v \frac{\partial v}{\partial y}\right)=-\frac{\partial p}{\partial y}+\delta^{4} \frac{\partial^{2} v}{\partial x^{2}}+\delta^{2} \frac{\partial^{2} v}{\partial y^{2}}-\delta^{2} \frac{v}{k}-\delta \frac{m M(u+1)}{\left(m^{2}+1\right)}-\delta^{2} \frac{M v}{\left(m^{2}+1\right)} \\
\delta R \operatorname{Pr}\left(u \frac{\partial \theta}{\partial x}+v \frac{\partial \theta}{\partial y}\right)=\delta^{2} \frac{\partial^{2} \theta}{\partial x^{2}}+\frac{\partial^{2} \theta}{\partial y^{2}}+\beta
\end{gathered}
$$

Assuming that the wave length is long and since the Reynolds number is low, then under this assumption (2.9)-(2.11) will take the following forms after dropping terms of order $\delta$ and higher:

$$
\begin{gathered}
0=-\frac{\partial p}{\partial x}+\frac{\partial^{2} u}{\partial y^{2}}-\frac{(u+1)}{k}+\operatorname{Gr} \theta-\frac{M(u+1)}{m^{2}+1}, \\
0=-\frac{\partial p}{\partial y} \\
0=\frac{\partial^{2} \theta}{\partial y^{2}}+\beta .
\end{gathered}
$$

The corresponding boundary conditions are

$$
\begin{array}{lll}
u=-1, & \theta=0 & \text { at } y=h_{1} \\
u=-1, & \theta=1 & \text { at } y=h_{2}
\end{array}
$$

where

$$
\begin{gathered}
h_{1}=1+a \cos 2 \pi x, \\
h_{2}=-d-b \cos (2 \pi x+\phi),
\end{gathered}
$$

and $a, b, d$, and $\phi$ satisfy the relation

$$
a^{2}+b^{2}+2 a b \cos \phi \leq(1+d)^{2}
$$




\section{Rate of Volume Flow}

The volume flow rate in wave frame of reference is given by

$$
q=\int_{h_{2}(x)}^{h_{1}(x)} u(x, y) d y
$$

where $h_{1}, h_{2}$ are functions of $x$ alone.

The instantaneous volume flow rate in the fixed frame is given by

$$
Q=\int_{H_{2}(x, t)}^{H_{1}(x, t)}[U(x, y, t)] d y=\int_{h_{2}(x)}^{h_{1}(x)}[u(x, y)+c] d y=q+c h_{1}-c h_{2} .
$$

The time-mean flow over time period $T$ at a fixed position $x$ is given by

$$
\bar{Q}(x, t)=\frac{1}{T} \int_{0}^{T} Q(x, y) d t
$$

Using (3.2) and (3.3) we get

$$
\bar{Q}(x, t)=q+c_{1} d_{1}+c_{2} d_{2} .
$$

On defining the dimensionless mean flow $\Theta$ in laboratory frame and $F$ in the wave frame,

$$
\Theta=\frac{\bar{Q}}{c d_{1}}, \quad F=\frac{q}{c d_{1}} .
$$

Using (3.4) and (3.5) we obtain

$$
\Theta=F+1+d,
$$

in which

$$
F=\int_{h_{2}(x)}^{h_{1}(x)} u(x, y) d y
$$

\section{Solution of the Problem}

The exact solutions of the set of (2.12) subject to the boundary conditions (2.13) and (2.14) are

$$
\begin{gathered}
\theta=\frac{1}{2\left(h_{2}-h_{1}\right)}\left\{-\beta\left(h_{2}-h_{1}\right) y^{2}+\left[2+\beta\left(h_{2}^{2}-h_{1}^{2}\right)\right] y+\beta h_{1} h_{2}\left(h_{1}-h_{2}\right)-2 h_{1}\right\}, \\
u=A \cosh \Gamma y+B \sinh \Gamma y+S_{1} y^{2}+S_{2} y+S_{3}-\frac{1}{\Gamma^{2}} \frac{d p}{d x}
\end{gathered}
$$


where

$$
\begin{aligned}
& \Gamma=\sqrt{\frac{\left(1+m^{2}\right)+k M}{k\left(1+m^{2}\right)}}, \quad S_{1}=\frac{-\beta \mathrm{Gr}}{2 \Gamma^{2}}, \quad S_{2}=\frac{\mathrm{Gr}}{\Gamma^{2}}\left(\frac{2+\beta\left(h_{2}^{2}-h_{1}^{2}\right)}{2\left(h_{2}-h_{1}\right)}\right), \\
& S_{3}=\frac{-1}{\Gamma^{2}}\left\{\frac{\mathrm{Gr}\left[\beta h_{1} h_{2}\left(h_{2}-h_{1}\right)+2 h_{1}\right]}{2\left(h_{2}-h_{1}\right)}+\frac{\beta \mathrm{Gr}}{\Gamma^{2}}+\Gamma^{2}\right\}, \\
& S_{4}=\cosh \Gamma h_{1} \sinh \Gamma h_{2}-\cosh \Gamma h_{2} \sinh \Gamma h_{1}, \\
& S_{5}=S_{1}\left(h_{2}^{2} \sinh \Gamma h_{1}-h_{1}^{2} \sinh \Gamma h_{2}\right), \\
& S_{6}=S_{2}\left(h_{2} \sinh \Gamma h_{1}-h_{1} \sinh \Gamma h_{2}\right), \\
& S_{7}=\sinh \Gamma h_{1}-\sinh \Gamma h_{2}, \\
& S_{8}=S_{1}\left(h_{1}^{2} \cosh \Gamma h_{2}-h_{2}^{2} \cosh \Gamma h_{1}\right), \\
& S_{9}=S_{2}\left(h_{1} \cosh \Gamma h_{2}-h_{2} \cosh \Gamma h_{1}\right), \\
& S_{10}=\cosh \Gamma h_{2}-\cosh \Gamma h_{1}, \\
& A=A_{1}+A_{2} \frac{d p}{d x}, \quad B=B_{1}+B_{2} \frac{d p}{d x}, \\
& A_{1}=\frac{S_{5}+S_{6}+S_{7}+S_{3} S_{7}}{S_{4}}, \quad A_{2}=\frac{-S_{7}}{S_{4} \Gamma^{2}}, \\
& B_{1}=\frac{S_{8}+S_{9}+S_{10}+S_{3} S_{10}}{S_{4}}, \quad B_{2}=\frac{-S_{10}}{S_{4} \Gamma^{2}},
\end{aligned}
$$

From (3.7), (4.2), and (4.12) we have

$$
F=\frac{1}{\Gamma}\left(A_{1} S_{7}-B_{1} S_{10}\right)+S_{11}+S_{12} \frac{d p}{d x},
$$

and therefore we can say that

$$
\frac{d p}{d x}=\frac{F-(1 / \Gamma)\left(A_{1} S_{7}-B_{1} S_{10}\right)-S_{11}}{S_{12}}
$$



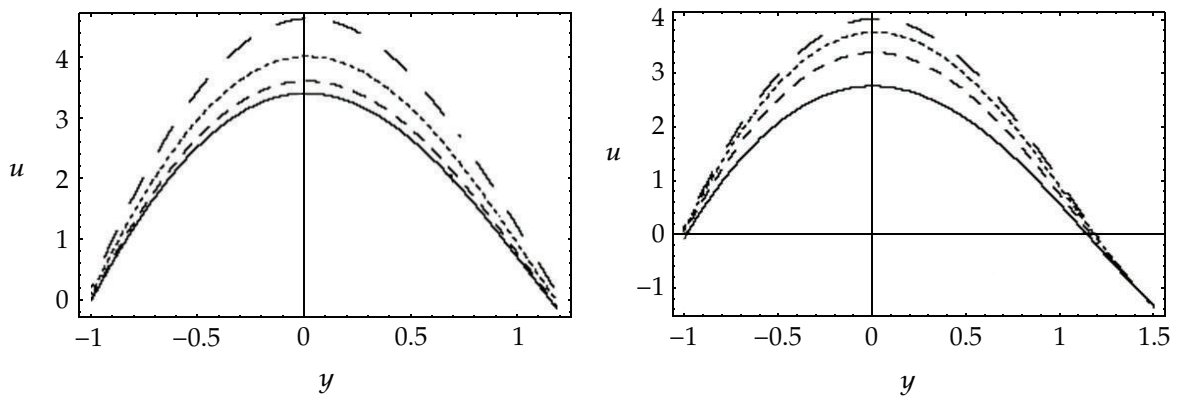

$$
\begin{array}{ll}
-m=0 & \ldots \ldots m=1 \\
--m=0.5 & --m=2
\end{array}
$$

(a)

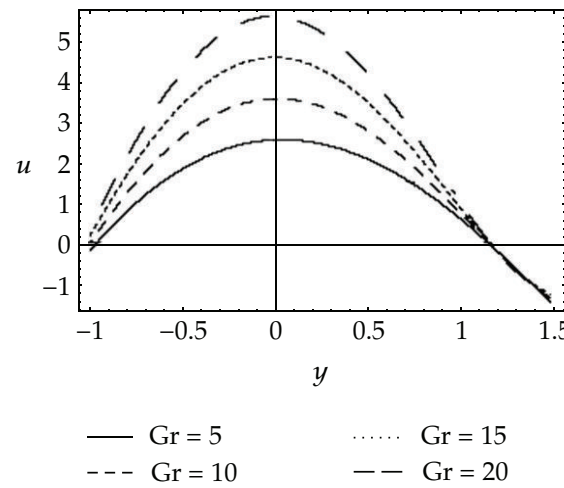

(c)

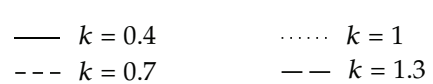

(b)

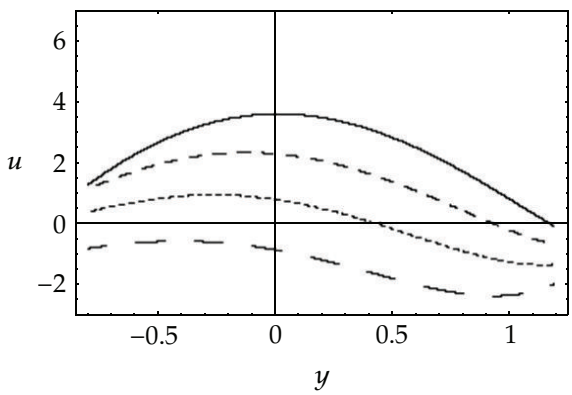

$$
\begin{aligned}
-d & =1 \\
-- & d=1.2
\end{aligned}
$$$$
\text { ….. } d=1.4
$$

(d)

Figure 2: The variation of the velocity distribution $u$ with $y$ for various values of Hall parameter $m$ (a), porosity parameter $k(\mathrm{~b})$, Grashof number $\mathrm{Gr}(\mathrm{c})$, and width of the channel $d(\mathrm{~d})$. The other parameters chosen are: $\Theta=4, x=0, d=1, M=1.2, a=0.4, b=0.2, \beta=2, k=1, \phi=\pi / 4$, Gr $=10$ (a); $\Theta=4, x=0$, $d=1, M=1.2, a=0.4, b=0.2, \beta=2, m=0.5, \phi=\pi / 4, \mathrm{Gr}=10(\mathrm{~b}) ; \Theta=4, x=0, d=1, M=1.2, a=0.4$, $b=0.2, \beta=2, m=0.5, \phi=\pi / 4, k=1$ (c); $\Theta=4, x=0, \beta=2, M=1.2, a=0.4, b=0.2, \mathrm{Gr}=10, m=0.5$, $\phi=\pi / 4, k=1(\mathrm{~d})$.

where

$$
\begin{gathered}
S_{11}=\left(h_{1}-h_{2}\right)\left\{\frac{S_{1}}{3}\left(h_{1}^{2}+h_{1} h_{2}+h_{2}^{2}\right)+\frac{S_{2}}{2}\left(h_{1}+h_{2}\right)+S_{3}\right\}, \\
S_{12}=\frac{A_{2} S_{7}}{\Gamma}-\frac{B_{2} S_{10}}{\Gamma}-\frac{\left(h_{1}-h_{2}\right)}{\Gamma^{2}} .
\end{gathered}
$$

The stream function $\psi$ can be obtained as follows:

$$
\psi=\int u d y
$$




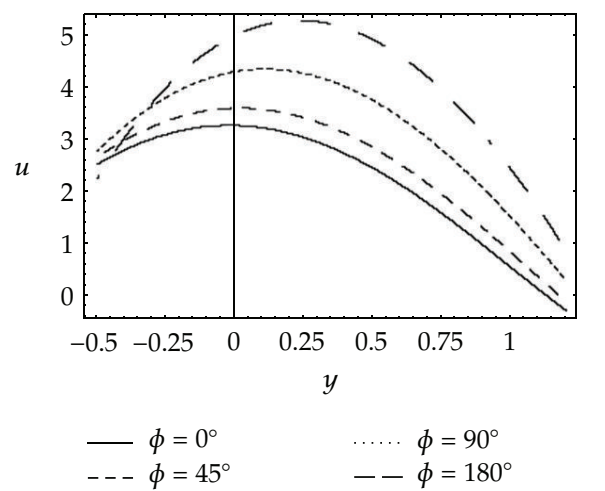

(a)

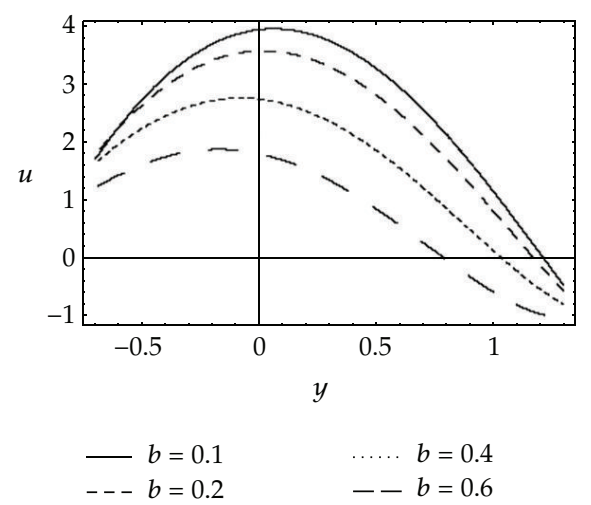

(c)
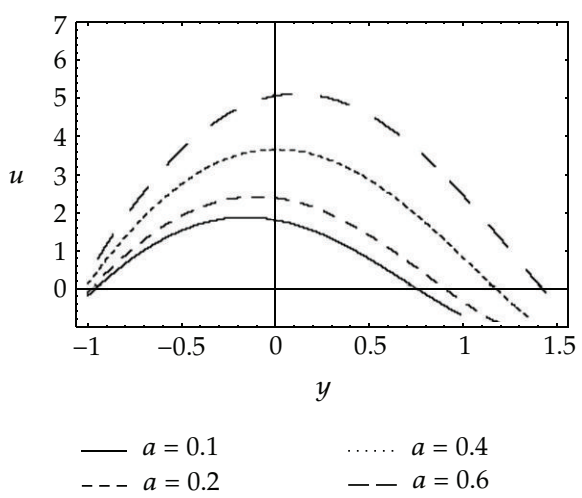

(b)

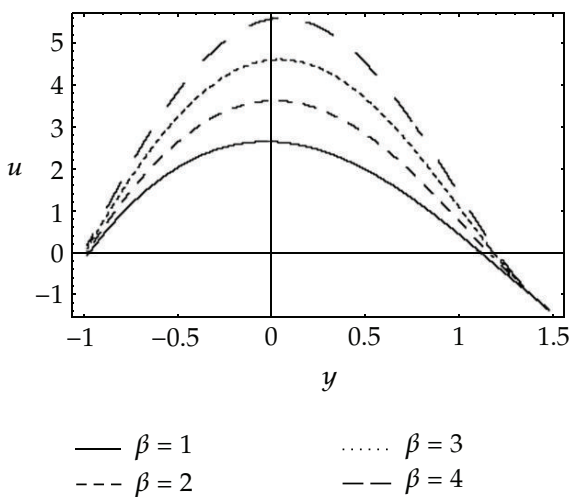

(d)

Figure 3: Variation of velocity distribution $u$ with $y$ for various values of phase angle $\phi$ (a), amplitude of the right wave $a(\mathrm{~b})$, amplitude of the left wave $b(\mathrm{c})$, and nondimensional heat source/sink parameter $\beta$ (d). The other parameters chosen are $\Theta=4, x=0, d=1, M=1.2, a=0.4, b=0.2, \beta=2, k=1, m=0.5$, $\mathrm{Gr}=10(\mathrm{a}) ; \Theta=4, x=0, d=1, M=1.2, k=1, b=0.2, \beta=2, m=0.5, \phi=\pi / 4, \mathrm{Gr}=310(\mathrm{~b}) ; \Theta=4, x=0$, $d=1, M=1.2, a=0.4, \mathrm{Gr}=10, \beta=2, m=0.5, \phi=\pi / 4, k=1$ (c); $\Theta=4, x=0, d=1, M=1.2, a=0.4$, $b=0.2, \mathrm{Gr}=10, m=0.5, \phi=\pi / 4, k=1(\mathrm{~d})$.

Using (4.2) and (4.12) we can easily get

$$
\begin{aligned}
\psi= & \frac{1}{\Gamma}\left(A_{1} \sinh \Gamma y+B_{1} \cosh \Gamma y\right)+\frac{1}{3} S_{1} y^{3}+\frac{1}{2} S_{2} y^{2}+S_{3} y \\
& +\left[\frac{-1}{\Gamma^{2}} y+\frac{A_{2}}{\Gamma} \sinh \Gamma y+\frac{B_{2}}{\Gamma} \cosh \Gamma y\right] \frac{d p}{d x} .
\end{aligned}
$$

The nondimensional expression for the pressure rise per wavelength is given by

$$
\Delta p_{\lambda}=\int_{0}^{1} \frac{\partial p}{\partial x} d x
$$



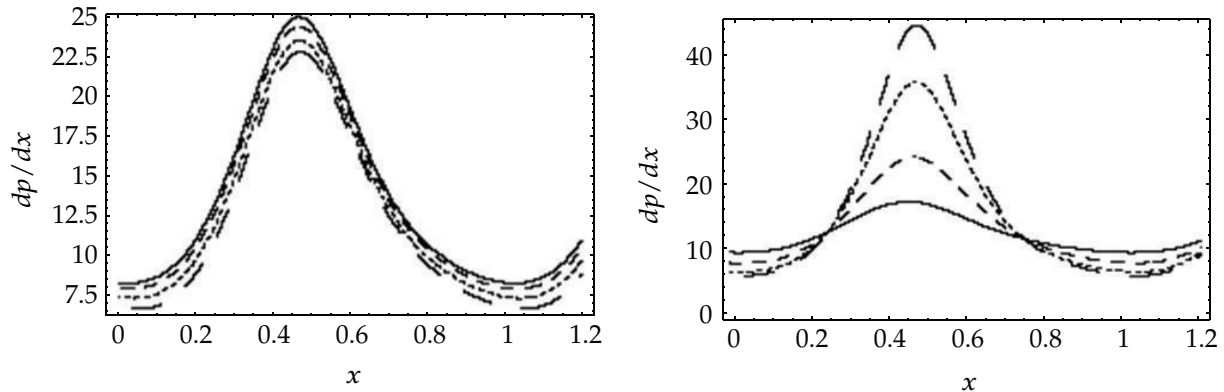

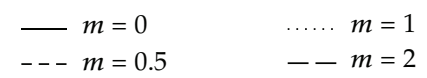

(a)

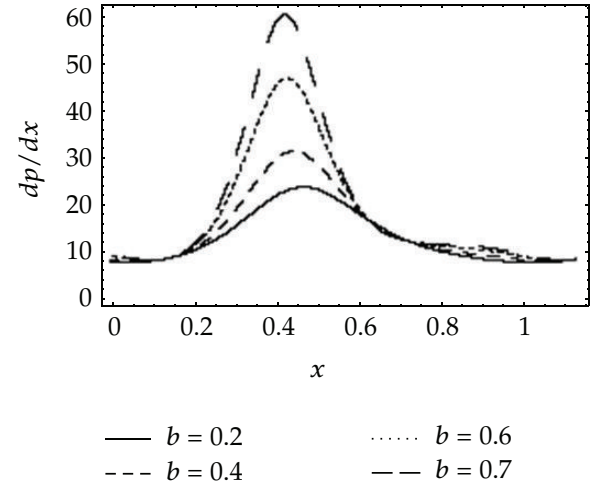

(c)

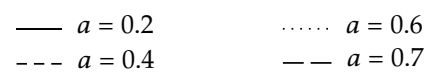

(b)

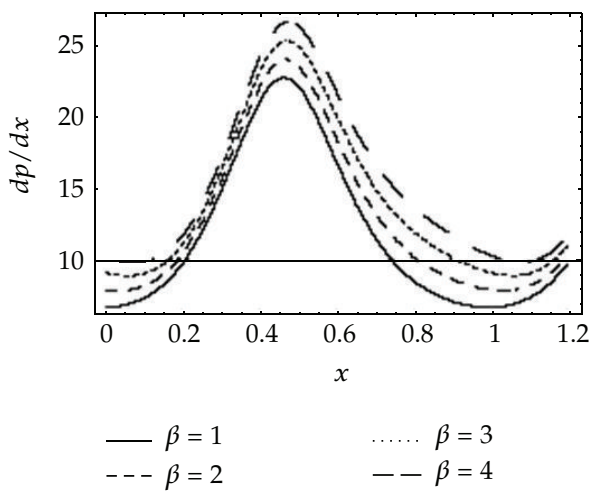

(d)

Figure 4: Distribution of pressure gradient $d p / d x$ with $x$ for different values of Hall parameter $m$ (a), amplitudes of right wave $a(\mathrm{~b})$, left wave $b(\mathrm{c})$, and the nondimensional heat source/sink parameter $\beta$ (d). The other parameters chosen are $F=-5, y=1, d=1, M=1.2, a=0.4, b=0.2, \beta=2, k=1, \phi=\pi / 4$, $\mathrm{Gr}=3(\mathrm{a}) ; F=-5, y=1, d=1, M=1.2, m=0.5, b=0.2, \beta=2, k=1, \phi=\pi / 4, \mathrm{Gr}=3(\mathrm{~b}) ; F=-5, y=1$, $d=1, M=1.2, m=0.5, a=0.4, \beta=2, k=1, \phi=\pi / 4, \mathrm{Gr}=3$ (c); $F=-5, y=1, d=1, M=1.2, m=0.5$, $a=0.4, b=0.2, k=1, \phi=\pi / 4, \mathrm{Gr}=3(\mathrm{~d})$.

The coefficient of heat transfer at the wall is given by

$$
Z=h_{2 x} \theta_{y} .
$$

Using (2.16) and (4.1) we get

$$
Z=2 \pi b \sin (2 \pi x+\phi)\left[-\beta y+\frac{2+\beta\left(h_{2}^{2}-h_{1}^{2}\right)}{2\left(h_{2}-h_{1}\right)}\right] .
$$

The nondimensional shear stress can be calculated as

$$
\tau=\frac{\tau^{*}}{\left(\mu c / d_{1}\right)}=\frac{\partial^{2} \psi}{\partial y^{2}}
$$



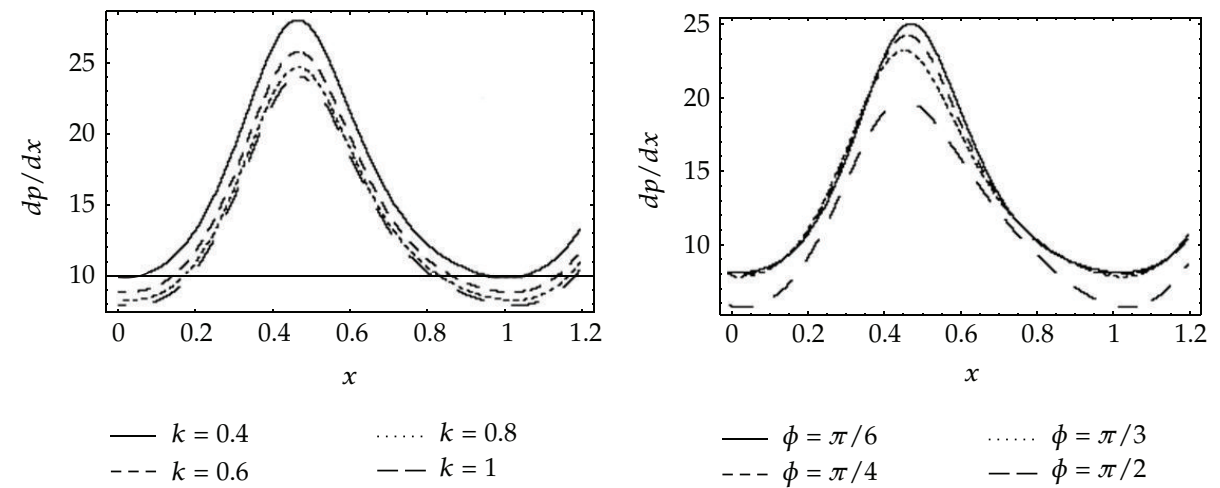

(a)

(b)

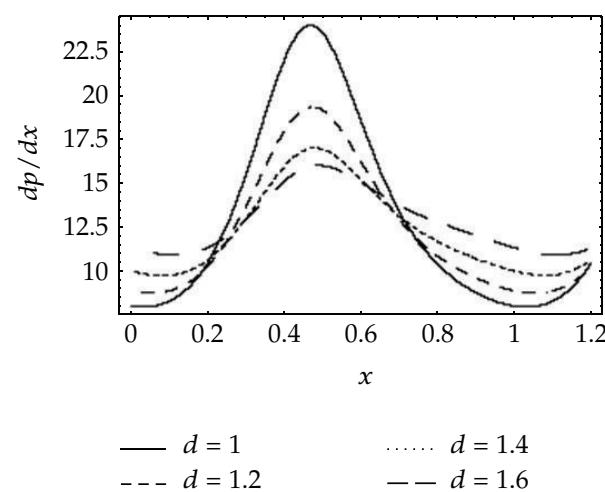

(c)

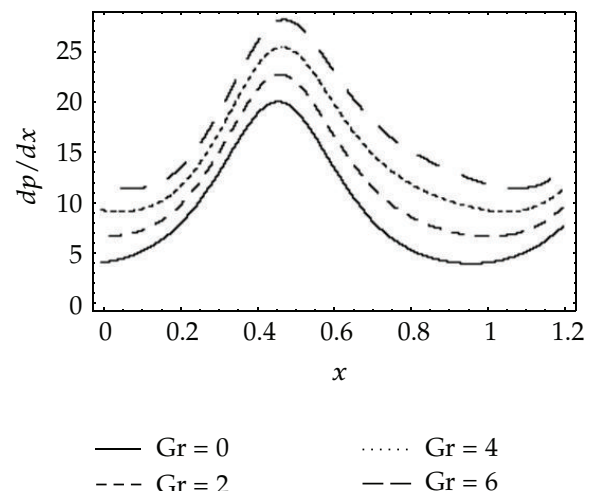

(d)

Figure 5: The distribution of the pressure gradient $d p / d x$ with $x$ for different values of porosity parameter $k(\mathrm{a})$, phase angle $\phi(\mathrm{b})$, width of the channel $d(\mathrm{c})$, and Grashof number $\mathrm{Gr}(\mathrm{d})$. The other parameters chosen are $F=-5, y=1, d=1, M=1.2, a=0.4, b=0.2, \beta=2, m=0.5, \phi=\pi / 4, \mathrm{Gr}=3$ (a); $F=-5, y=1$, $d=1, M=1.2, a=0.4, b=0.2, \beta=2, m=0.5, k=1, \mathrm{Gr}=3(\mathrm{~b}) ; F=-5, y=1, k=1, M=1.2, a=0.4$, $b=0.2, \beta=2, m=0.5, \phi=\pi / 4, \mathrm{Gr}=3$ (c); $F=-5, y=1, d=1, M=1.2, a=0.4, b=0.2, \beta=2, m=0.5$, $\phi=\pi / 4, k=1(\mathrm{~d})$.

Substituting from (4.20) and (4.12) into (4.24) we get

$$
\tau=\Gamma(A \sinh \Gamma y+B \cosh \Gamma y)+2 S_{1} y+S_{2} .
$$

The friction forces $F_{\lambda i}$ at the walls of the channel are given by

$$
F_{\lambda_{i}}=\int_{0}^{1} h_{i}\left(-\frac{\partial p}{\partial x}\right) d x, \quad i=1,2
$$

\section{Results and Discussion}

This section represents the graphical results in order to be able to discuss the quantitative effects of the sundry parameters involved in the analysis. The variation of velocity distribution with $y$ for various values of $m, k, \mathrm{Gr}$, and $\beta$ is studied in Figure 2. Figure 2(a) 

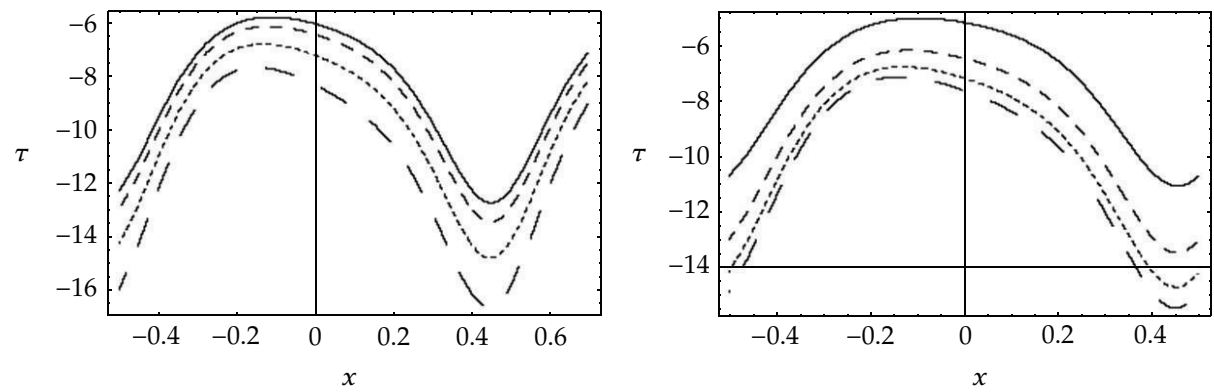

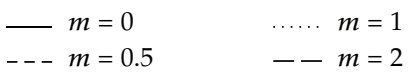

(a)

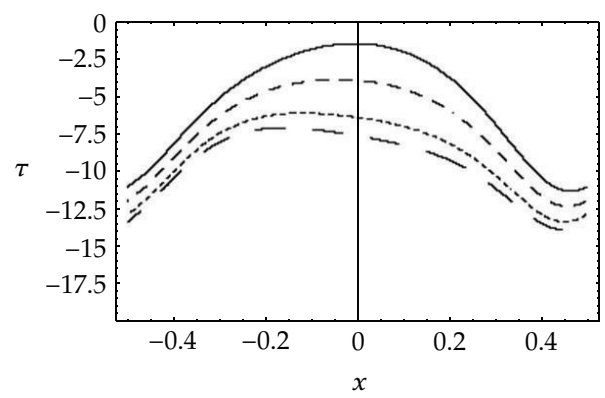

$\begin{aligned}-\beta & =-2 \\ --\beta & =0\end{aligned}$

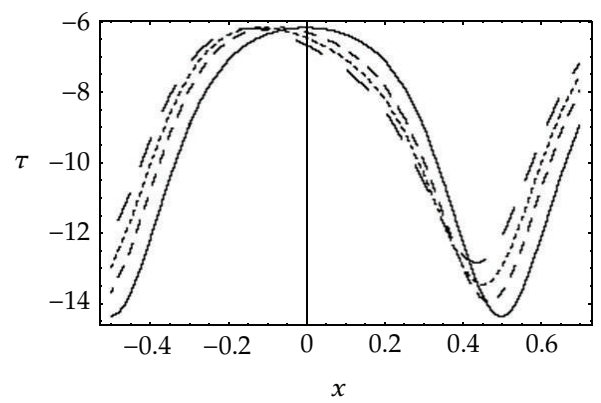

$\begin{aligned} \phi & =0 \\ \ldots & =\pi / 6\end{aligned}$ (c)

… $\beta=2$

$--\beta=3$

..... $\phi=\pi / 4$

(e)

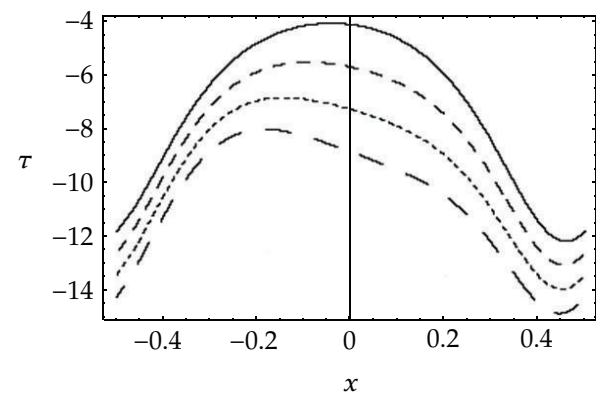

- $\mathrm{Gr}=0$

$---\mathrm{Gr}=2$

… $\mathrm{Gr}=4$

$--\mathrm{Gr}=6$

(d)

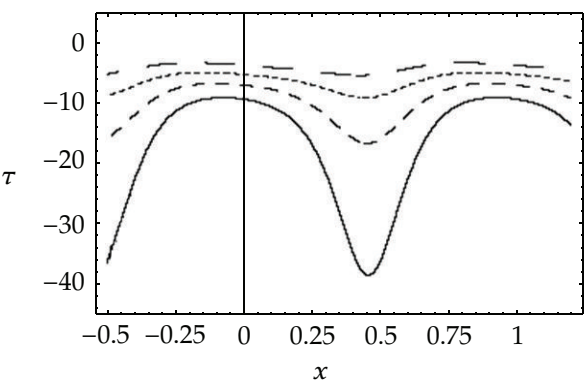

$-d=0.1$

..... $d=0.7$

$---d=0.4$

(f)

Figure 6: The shear stress distribution at the right wall with $x$ for different values of Hall parameter $m$ (a), porosity parameter $k(b)$, nondimensional heat source/sink parameter $\beta$ (c), Grashof number Gr (d), phase angle $\phi(\mathrm{e})$, and width of the channel $d(\mathrm{f})$. The other parameters chosen are $\Theta=3, d=0.5, M=1.2$, $a=0.4, b=0.2, \beta=2, k=1, \phi=\pi / 4, \mathrm{Gr}=3(\mathrm{a}) ; \Theta=3, d=0.5, M=1.2, a=0.4, b=0.2, \beta=2, m=0.5$, $\phi=\pi / 4, \mathrm{Gr}=3(\mathrm{~b}) ; \Theta=3, d=0.5, M=1.2, a=0.4, b=0.2, m=0.5, k=1, \phi=\pi / 4, \mathrm{Gr}=(\mathrm{c}) ; \Theta=3$, $d=0.5, M=1.2, a=0.4, b=0.2, \beta=2, k=1, \phi=\pi / 4, m=0.5(\mathrm{~d}) ; \Theta=3, d=0.5, M=1.2, a=0.4, b=0.2$, $\beta=2, k=1, m=0.5, \mathrm{Gr}=3(\mathrm{e}) ; \Theta=3, m=0.5, M=1.2, a=0.4, b=0.2, \beta=2, k=1, \phi=\pi / 4, \mathrm{Gr}=3$ (f). 

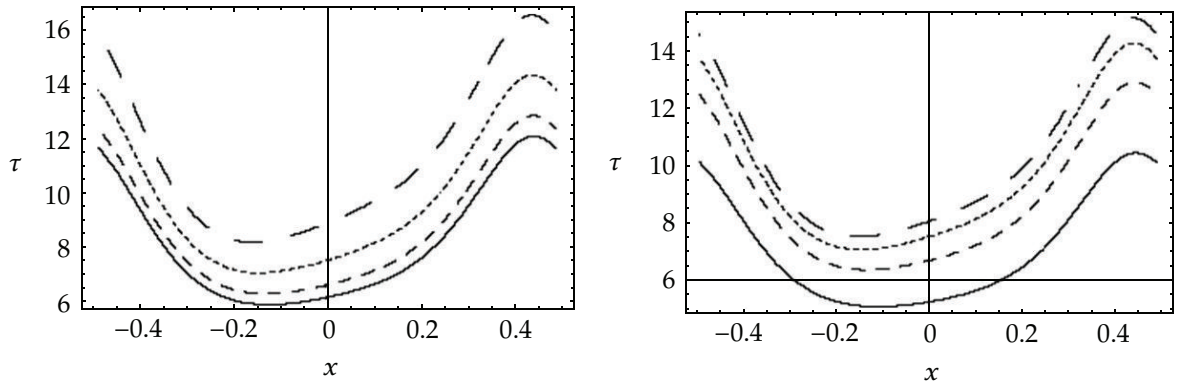

$$
\begin{aligned}
-m & =0 \\
--m & =0.5
\end{aligned}
$$

(a)

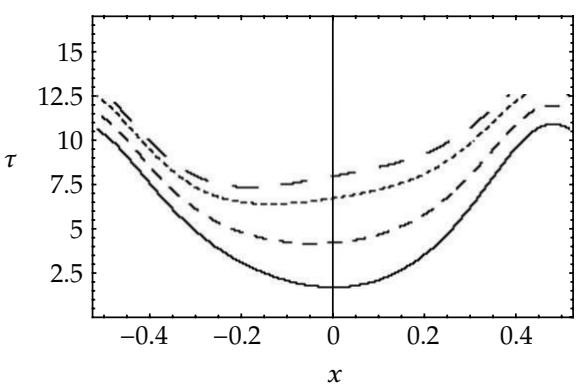

$$
\begin{aligned}
-\beta & =-2 \\
--\beta & =0
\end{aligned}
$$$$
\begin{aligned}
& \ldots \beta=2 \\
& --\beta=3
\end{aligned}
$$

(c)

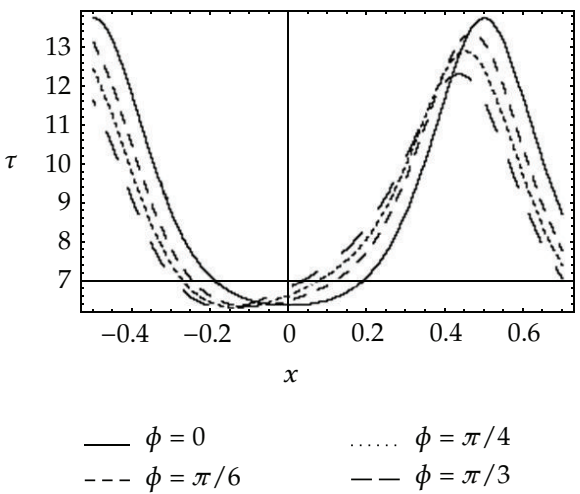

(e)

$$
\begin{aligned}
k & =0.5 & \ldots \ldots k & =1.5 \\
--k & =1 & --k & =2
\end{aligned}
$$

(b)

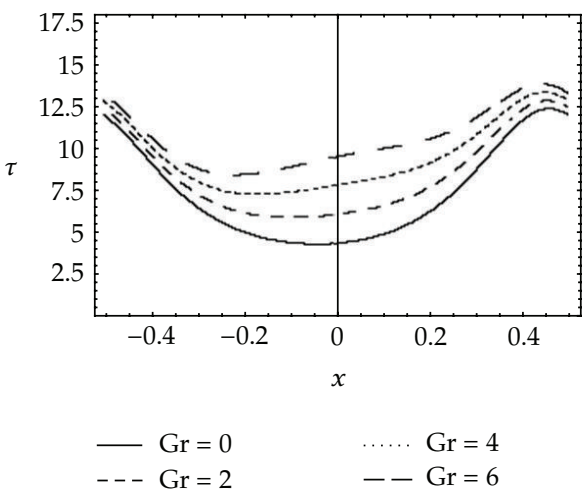

(d)

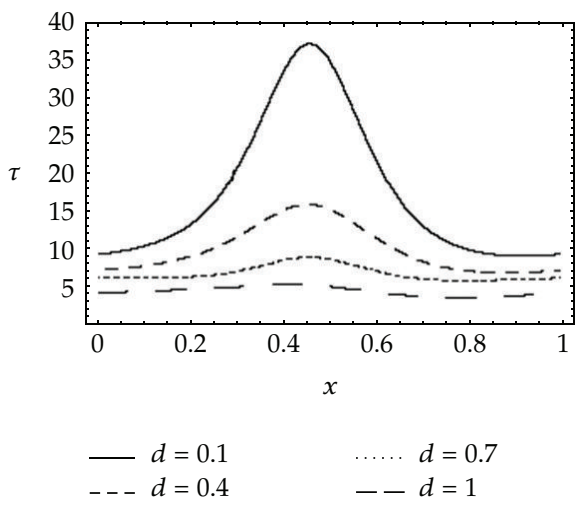

(f)

Figure 7: The shear stress distribution at the left wall with $x$ for different values of Hall parameter $m$ (a), porosity parameter $k(b)$, nondimensional heat source/sink parameter $\beta(\mathrm{c})$, Grashof number $\mathrm{Gr}(\mathrm{d})$, phase angle $\phi(\mathrm{e})$, and width of the channel $d$ (f). The other parameters chosen are $\Theta=3, d=0.5, M=1.2$, $a=0.4, b=0.2, \beta=2, k=1, \phi=\pi / 4, \mathrm{Gr}=3(\mathrm{a}) ; \Theta=3, d=0.5, M=1.2, a=0.4, b=0.2, \beta=2, m=0.5$, $\phi=\pi / 4, \mathrm{Gr}=3(\mathrm{~b}) ; \mathrm{\Theta}=3, d=0.5, M=1.2, a=0.4, b=0.2, m=0.5, k=1, \phi=\pi / 4, \mathrm{Gr}=3$ (c); $\Theta=3$, $d=0.5, M=1.2, a=0.4, b=0.2, \beta=2, k=1, \phi=\pi / 4, m=0.5(\mathrm{~d}) ; \Theta=3, d=0.5, M=1.2, a=0.4, b=0.2$, $\beta=2, k=1, m=0.5, \mathrm{Gr}=3$ (e); $\Theta=3, m=0.5, M=1.2, a=0.4, b=0.2, \beta=2, k=1, \phi=\pi / 4, \mathrm{Gr}=3$ (f). 


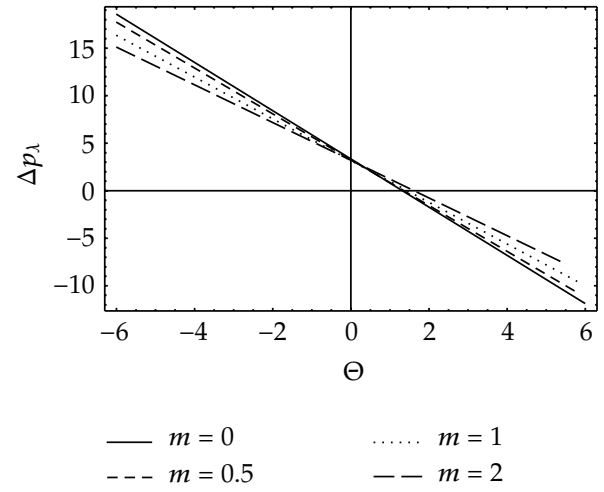

(a)

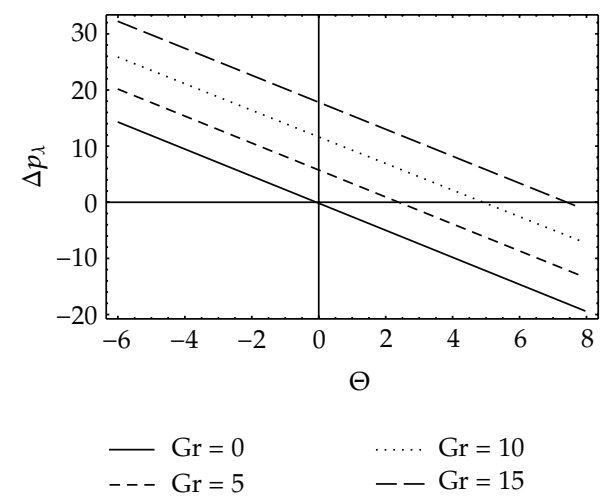

(c)

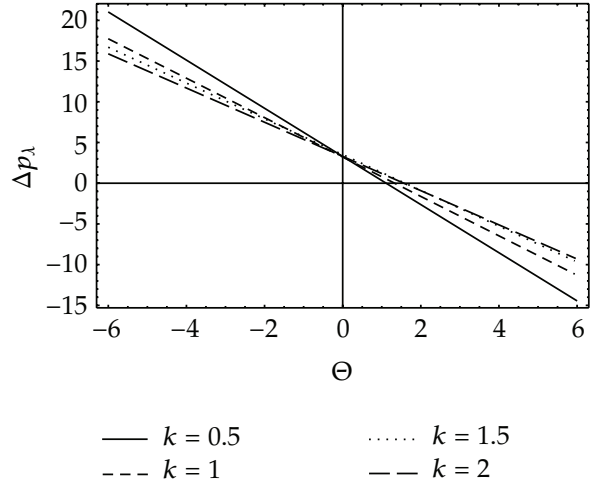

(b)

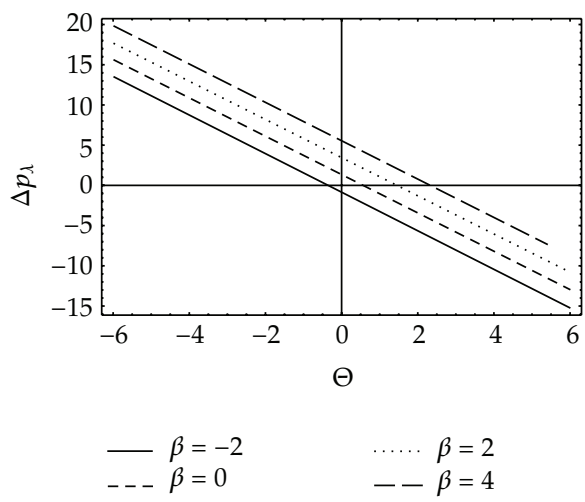

(d)

Figure 8: The variation of pressure rise $\Delta p_{\lambda}$ with $\Theta$ for different values of Hall parameter $m$ (a), porosity parameter $k(b)$, Grashof number Gr (c), and nondimensional heat source/sink parameter $\beta(\mathrm{d})$. The other parameters chosen are $d=1, M=1.2, a=0.1, b=0.2, \phi=\pi / 2, k=1, \beta=2$, Gr $=3$ (a); $d=1, M=1.2$, $a=0.1, b=0.2, \phi=\pi / 2, m=0.5, \beta=2, \mathrm{Gr}=3(\mathrm{~b}) ; d=1, M=1.2, a=0.1, b=0.2, \phi=\pi / 2, k=1, m=0.5$, $\mathrm{Gr}=3(\mathrm{c}) ; d=1, M=1.2, a=0.1, b=0.2, \phi=\pi / 2, k=1, \beta=2, m=0.5(\mathrm{~d})$.

studies the effect of Hall parameter $m$ on the velocity $u$ and it is noticed that the velocity increases by increasing $m$. Also, it is noticed that increasing each of the porosity parameter $k$ and Grashof number Gr led to an increase in the velocity as shown in Figures 2(b) to 2(c), but the velocity decreases by increasing the width of the channel d as shown in Figure 2(d).

Figure 3 illustrates the effects of phase angle $\phi$, amplitudes of the right and left waves $a, b$, respectively, and nondimensional heat source/sink parameter $\beta$ on velocity distribution. It reveals that the velocity increases by increasing each of phase angle $\phi$, amplitude of the right wave $a$, and nondimensional heat source/sink parameter $\beta$. On the other hand the velocity decreases by increasing the amplitude of the left wave $b$.

In Figures 4 and 5 we considered variation of the pressure gradient with $x$ for different values of $m, a, b$, and $\beta$ (Figure 4) and $k, \phi, d$, and Gr (Figure 5). It is clear from Figure 4 that the pressure gradient decreases by increasing the Hall parameter $m$ while it increases by increasing each of $a, b$, and $\beta$. Further it is observed from Figure 5 that the pressure gradient decreases by increasing each of $k, \phi$, and $d$, while increasing the Grashof number Gr led to increasing the pressure gradient. Figures 6 and 7 show the stress distribution at the right 


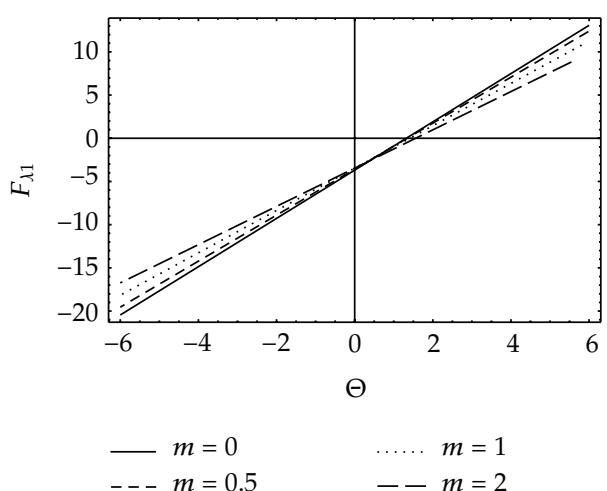

(a)

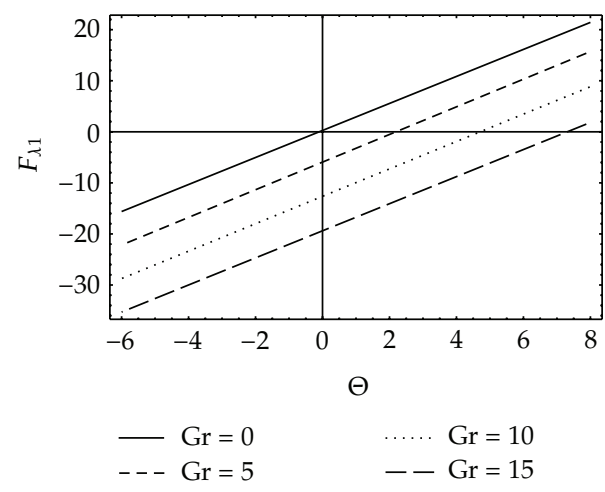

(c)

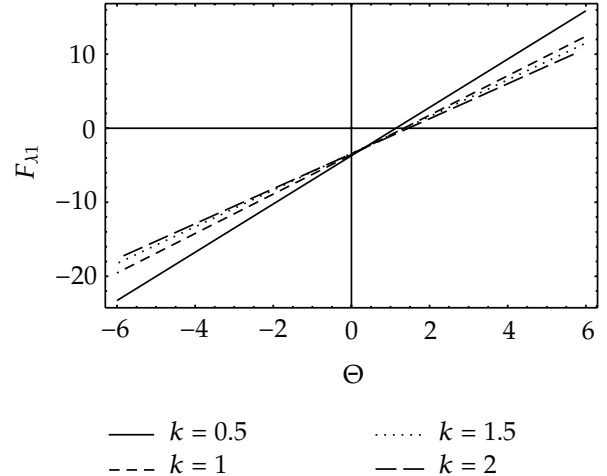

(b)

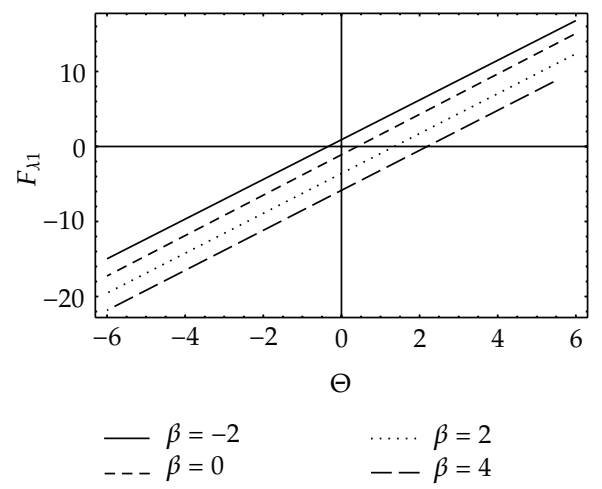

(d)

Figure 9: The variation of friction force at the right wall $F_{\lambda 1}$ with $\Theta$ for different values of Hall parameter $m$ (a), porosity parameter $k(\mathrm{~b})$, Grashof number $\mathrm{Gr}(\mathrm{c})$, and nondimensional heat source/sink parameter $\beta$ (d). The other parameters chosen are $d=1, M=1.2, a=0.1, b=0.2, \phi=\pi / 2, k=1, \beta=2$, Gr $=3$ (a); $d=1, M=1.2, a=0.1, b=0.2, \phi=\pi / 2, m=0.5, \beta=2, \mathrm{Gr}=3(\mathrm{~b}) ; d=1, M=1.2, a=0.1, b=0.2, \phi=\pi / 2$, $k=1, m=0.5, \mathrm{Gr}=3$ (c); $d=1, M=1.2, a=0.1, b=0.2, \phi=\pi / 2, k=1, \beta=2, m=0.5$ (d).

and left walls, respectively. It is obvious from Figure 6 that the amplitude of the shear stress decreases by increasing each of $m, k, \beta$, and Gr. Figure 6(e) indicates that the amplitude of the shear stress increases by increasing the phase angle $\phi$ and after a certain value of $x$ the situation is reversed. Figure 6(f) shows that increasing the width of the channel led to an increase in the shear stress at the right wall. Situation is in sharp contrast in Figure 7 we note that the shear stress at the left wall increases by increasing each of $m, k, \beta$, and Gr, also increasing $\phi$ led to a decrease in the shear stress, and after a certain value of $x$ the situation is reversed. Figure 7(f) reveals that the shear stress at the left wall decreases by increasing the width of the channel. Variation of the pressure rise per wavelength $\Delta p_{\lambda}$ with the mean flow $\Theta$ for different values $m, k, \mathrm{Gr}$, and $\beta$ is studied in Figure 8. From Figure 8(a) it is observed that, when $\Delta p_{\lambda}>5.12$, the pumping rate decreases by increasing $m$, further, it coincides each other between $2.1<\Delta p_{\lambda}<5.12$, and it increases by increasing $m$ when $\Delta p_{\lambda}<2.1$ (the free pumping $\Delta p_{\curlywedge}=0$ and copumping $\Delta p_{\curlywedge}<0$ occur in this region). Figure $8(\mathrm{~b})$ shows that when $\Delta p_{\curlywedge}>4.61$ the pumping rate decreases with an increase in $k$, when $1.97<\Delta p_{\lambda}<4.61$ the curves coincide with each other, and when $\Delta p_{\lambda}<1.97$ the pumping increases by increasing 


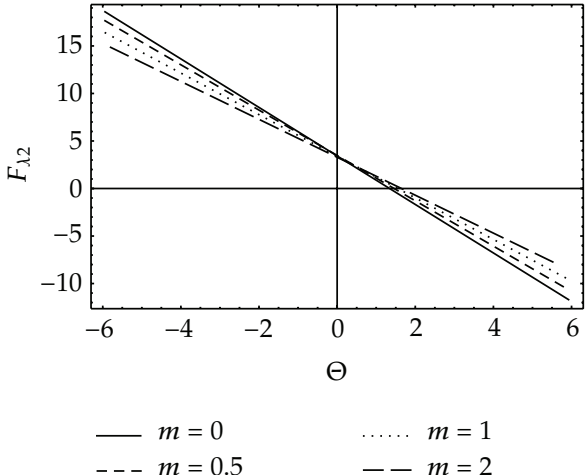

(a)

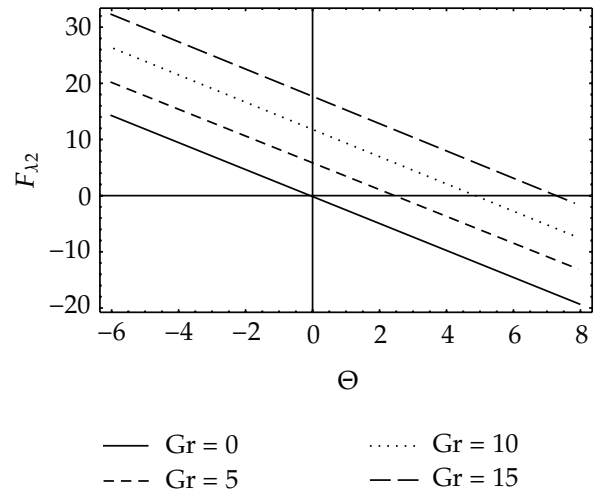

(c)

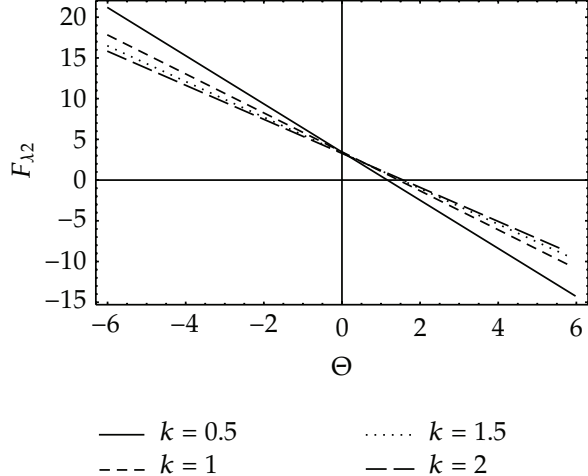

(b)

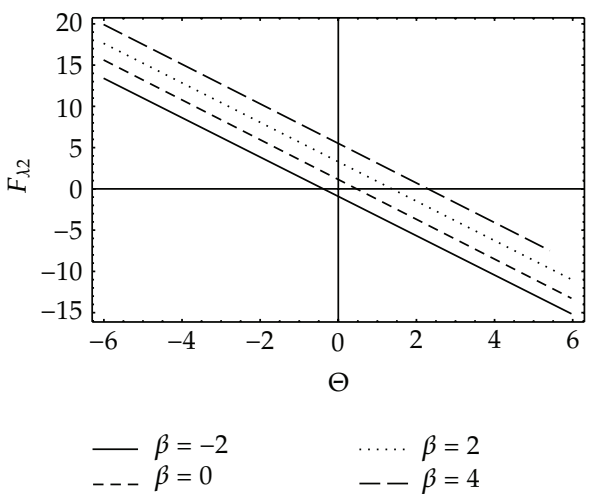

(d)

Figure 10: The variation of friction force at the left wall $F_{12}$ with $\Theta$ for different values of Hall parameter $m$ (a), porosity parameter $k(\mathrm{~b})$, Grashof number $\mathrm{Gr}(\mathrm{c})$, and nondimensional heat source/sink parameter $\beta$ (d). The other parameters chosen are $d=1, M=1.2, a=0.1, b=0.2, \phi=\pi / 2, k=1, \beta=2$, Gr $=3(\mathrm{a})$; $d=1, M=1.2, a=0.1, b=0.2, \phi=\pi / 2, m=0.5, \beta=2, \mathrm{Gr}=3(\mathrm{~b}) ; d=1, M=1.2, a=0.1, b=0.2, \phi=\pi / 2$, $k=1, m=0.5, \mathrm{Gr}=3$ (c); $d=1, M=1.2, a=0.1, b=0.2, \phi=\pi / 2, k=1, \beta=2, m=0.5$ (d).

$k$. The pumping rate increases by increasing each of $\mathrm{Gr}$ and $\beta$ as shown in Figures 8(c) and $8(d)$.

Figures 9 and 10 study the variation of friction forces $F_{\lambda 1}$ and $F_{\lambda 2}$ with $\Theta$ for different values of $m, k, \mathrm{Gr}$, and $\beta$. In Figure 9 (a) we notice that when $F_{\lambda 1}>-1.66$ the pumping rate decreases by increasing $m$, also when $-5.74<F_{\lambda 1}<-1.66$ the curves coincide with each other, and when $F_{\lambda 1}<-5.74$ the pumping rate increases by increasing $m$. Nearly the same situation occurs in Figure 9(b); when $F_{\lambda 1}>-1.85$ the pumping rate decreases by increasing $k$, when $-4.29<F_{\lambda 1}<-1.85$ the curves coincide with each other; and when $F_{\lambda 1}<-4.29$ the pumping rate increases by increasing $k$. Figures $9(\mathrm{c})$ and $9(\mathrm{~d})$ shows that pumping rate decreases by increasing each of $\mathrm{Gr}$ and $\beta$ for all values of $F_{\lambda 1}$. Figure 10(a) shows that when $F_{\lambda 2}>5.25$ the pumping rate decreases by increasing $m$ and curves coincide with each other for $1.97<F_{\lambda 2}<5.25$ and when $F_{\lambda 2}<-1.97$ the pumping rate increases by increasing $m$. From Figure $10(\mathrm{~b})$ we find that when $F_{\lambda 2}>4.73$ the pumping rate decreases with an increase in $k$ while it coincides each other between $1.88<F_{\lambda 2}<4.73$, also the pumping rate increases by 

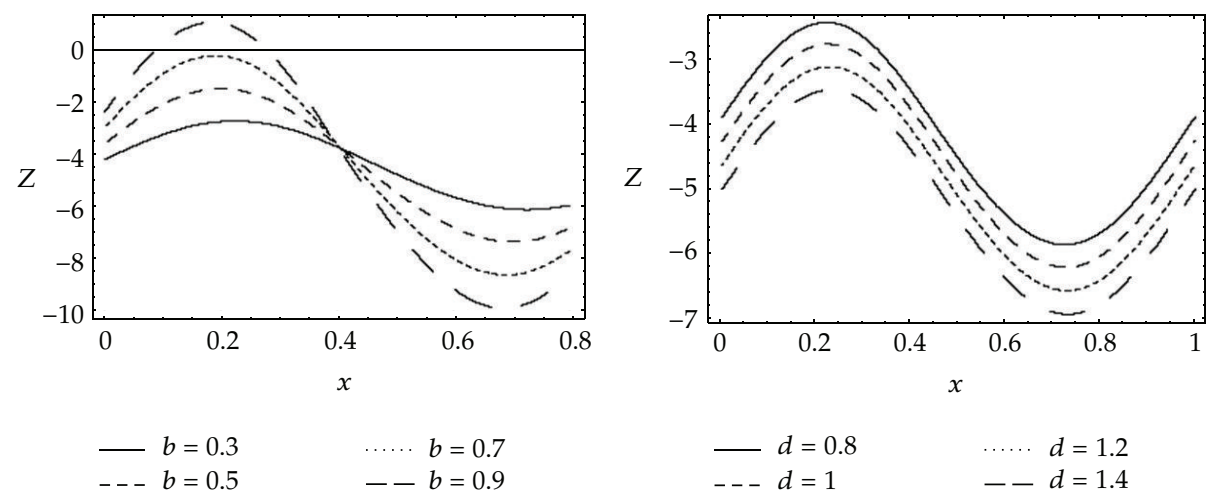

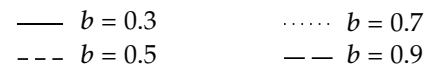

(a)

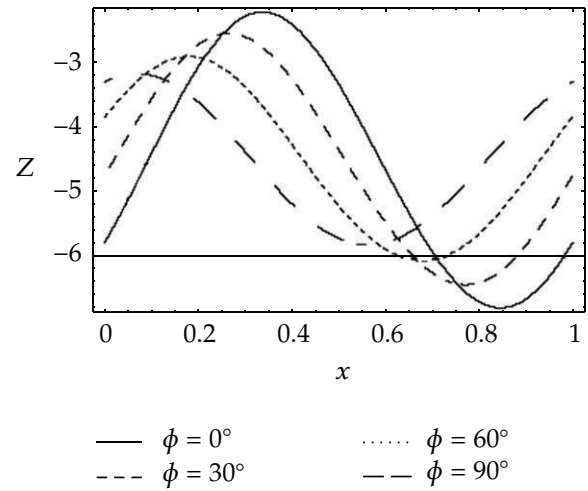

(c) (b)

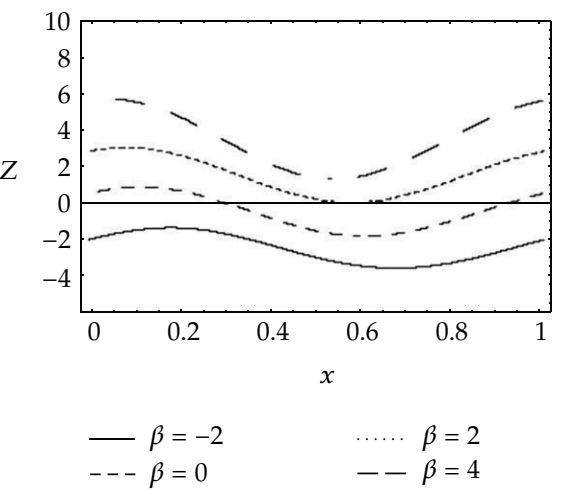

(d)

Figure 11: The variation of heat transfer coefficient $Z$ with $x$ for different values of amplitude of the left wave $b(\mathrm{a})$, channel width $d(\mathrm{~b})$, phase angle $\phi(\mathrm{c})$, and nondimensional heat source/sink parameter $\beta(\mathrm{d})$. The other parameters chosen are $d=1, a=0.4, \beta=-4, \phi=\pi / 4$ (a); $a=0.4, b=0.3, \beta=-4, \phi=\pi / 4$ (b); $b=0.3, a=0.4, \beta=-4, d=1$ (c); $d=1, a=0.4, \phi=\pi / 4, b=0.2$ (d).

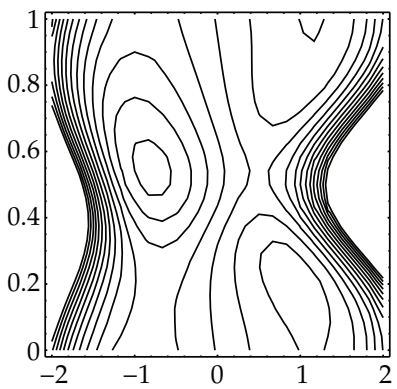

(a)

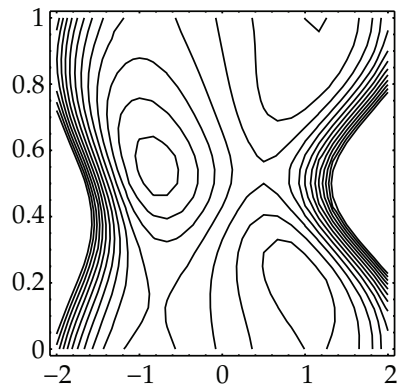

(b)

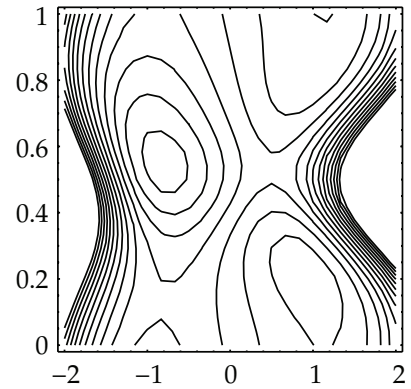

(c)

Figure 12: Streamlines for three different values of $m$ : (a) $m=1$, (b) $m=2$, (c) $m=3$. The other parameters chosen are $\Theta=4, d=1, M=1.2, a=0.4, b=0.2, \beta=-2, k=0.5, \phi=\pi / 4, \mathrm{Gr}=2$. 


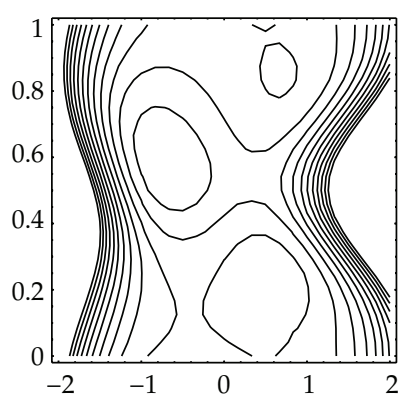

(a)

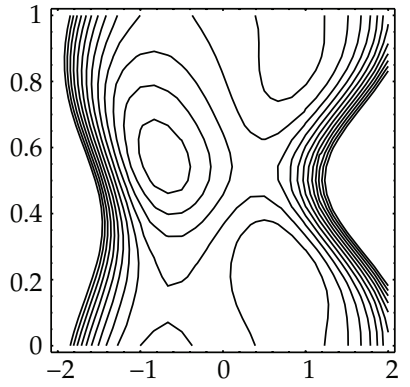

(b)

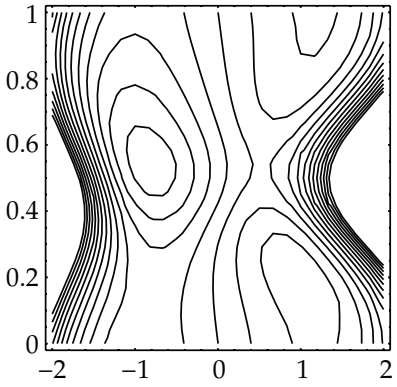

(c)

Figure 13: Streamlines for three different values of $\Theta$ : (a) $\Theta=2$, (b) $\Theta=2.5$, (c) $\Theta=5$. The other parameters chosen are $m=1.5, d=1, M=1.2, a=0.4, b=0.2, \beta=-2, k=0.5, \phi=\pi / 4, \mathrm{Gr}=2$.

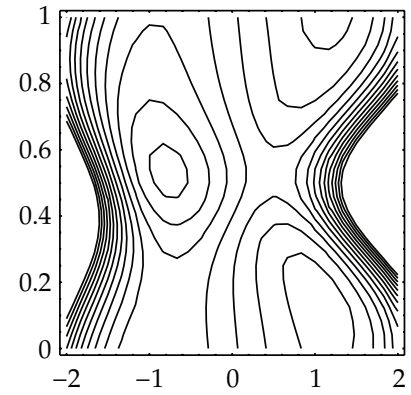

(a)

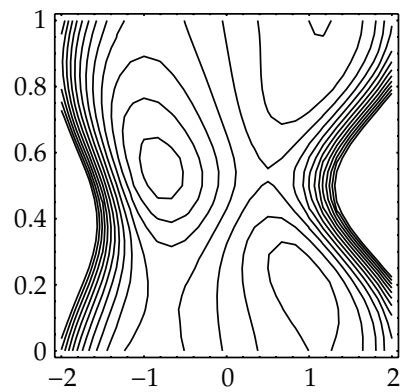

(b)

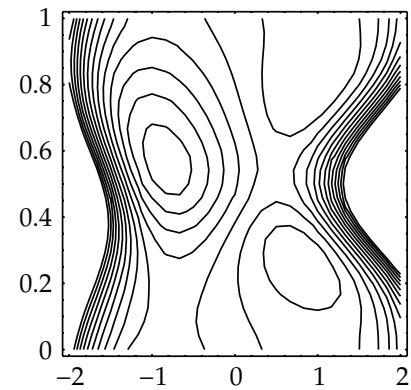

(c)

Figure 14: Streamlines for three different values of Gr: (a) Gr =0, (b) Gr = 2, (c) Gr $=5$. The other parameters chosen are $\Theta=4, d=1, M=1.2, a=0.4, b=0.2, \beta=-2, k=0.5, \phi=\pi / 4, m=1.5$.

increasing $k$ when $F_{\lambda 2}<1.88$. The pumping rate increases by increasing each of $\mathrm{Gr}$ and $\beta$ as shown in Figures 10(c) and 10(d).

The variation of heat transfer coefficient $Z$ with $x$ for different values $b, a, d$, and $\phi$ is studied in Figure 11. It is obvious from Figure 11(a) that increasing the amplitude of the left wave $b$ led to increasing the heat transfer coefficient and after a certain value of $x$ the situation is reversed; also from Figure 11(b) we notice that increasing the channel width decreases the heat transfer coefficient. The maximum value of heat transfer coefficient decreases by increasing $\phi$ as shown in Figure 11(c). Figure 11(d) shows that increasing $\beta$ produces an increase in heat transfer coefficient parameter.

\section{Trapping Phenomenon}

The formation of an internally circulating bolus of fluid is called trapping. The trapped bolus moves along with the wave. The effect of Hall parameter $m$ on trapping can be observed through Figure 12 where the size of bolus increases by increasing $m$. Figure 13 depicts that increasing $\Theta$ led to an increase in trapped bolus size and also increases the number of trapped bolus. In Figures 14 and 15 we notice that the size of trapped bolus increases by increasing 


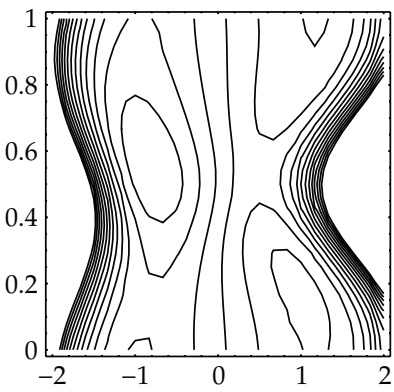

(a)

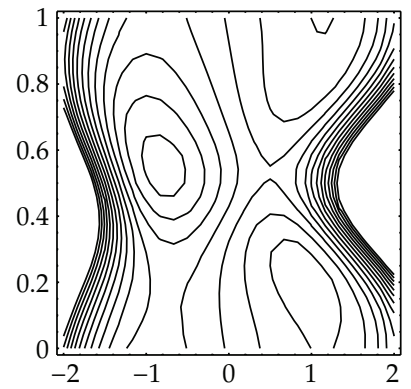

(b)

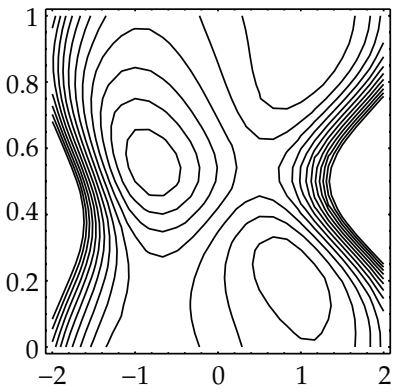

(c)

Figure 15: Streamlines for three different values of $k$ : (a) $k=0.2$, (b) $k=0.5$, (c) $k=0.8$. The other parameters chosen are $\Theta=4, d=1, M=1.2, a=0.4, b=0.2, \beta=-2, m=1.5, \phi=\pi / 4, \mathrm{Gr}=2$.

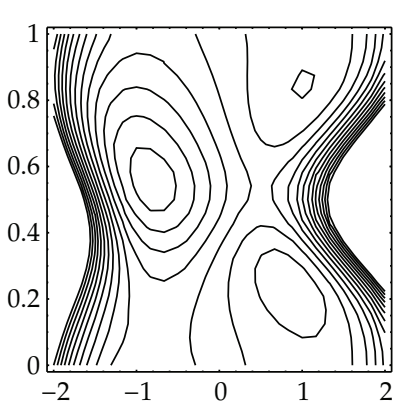

(a)

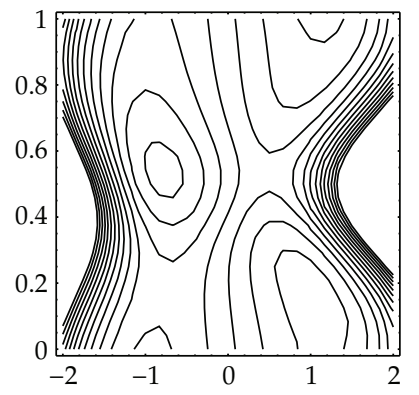

(b)

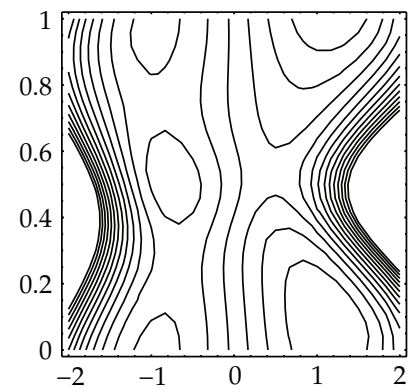

(c)

Figure 16: Streamlines for three different values of $\beta$ : (a) $\beta=-4$, (b) $\beta=0$, (c) $\beta=4$. The other parameters chosen are $\Theta=4, d=1, M=1.2, a=0.4, b=0.2, m=1.5, k=0.5, \phi=\pi / 4, \mathrm{Gr}=2$.

each of $\mathrm{Gr}$ and $k$. On the other hand the size of trapped bolus decreases by increasing $\beta$ as shown in Figure 16. The effects of $a, b$, and $d$ on trapping are studied in Figures 17, 18, and 19 , respectively. It is clear that the number of trapped bolus increases by increasing $a$ and decreases by increasing $b$. Furthermore, increasing the channel width led to an increase in size of trapped bolus. Figure 20 studies the case of symmetric channel $(a=b, d=1$, and $\phi=0)$. It is observed that the size of trapped bolus increases by increasing each of $m, k$, and $\Theta$ and decreases by increasing each of $\mathrm{Gr}$ and $\beta$.

\section{Conclusions}

In the present paper, the effects of Hall current and heat transfer on the peristaltic transport of a Newtonian fluid in an asymmetric channel through a porous medium under assumptions of a constant external magnetic field, low Reynolds number, and long wavelength are investigated. The governing equations are first modeled and then solved analytically. The effects of various emerging parameters on axial velocity, stream function, pressure gradient, friction forces, and shear stresses are observed from the graphs. The results are summarized as follows. 


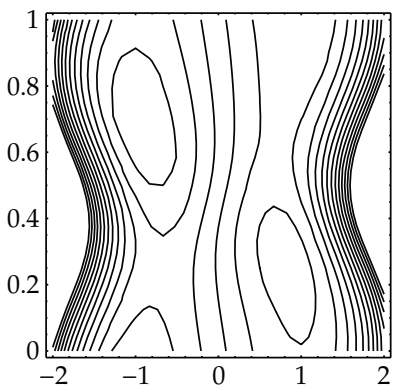

(a)

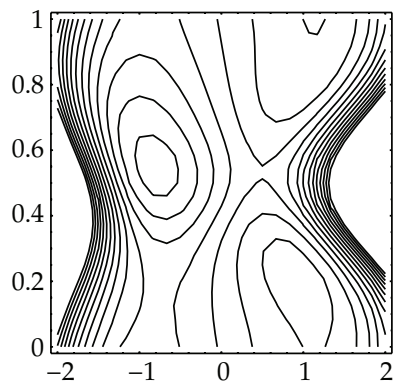

(b)

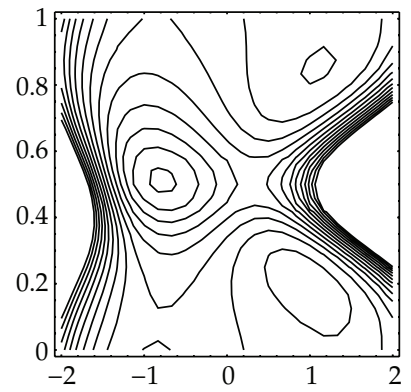

(c)

Figure 17: Streamlines for three different values of $a$ : (a) $a=0.2$, (b) $a=0.4$, (c) $a=0.6$. The other parameters chosen are $\Theta=4, d=1, M=1.2, m=1.5, b=0.2, \beta=-2, k=0.5, \phi=\pi / 4, \mathrm{Gr}=2$.

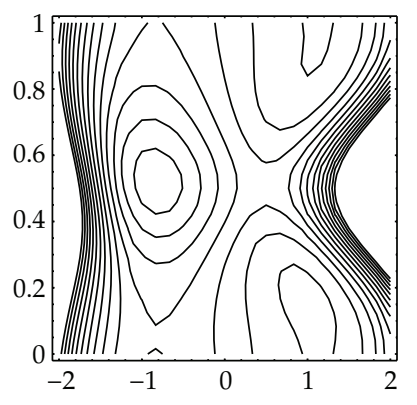

(a)

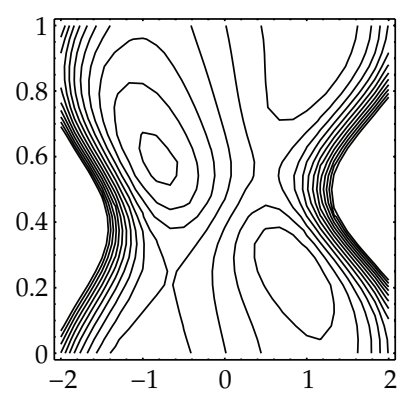

(b)

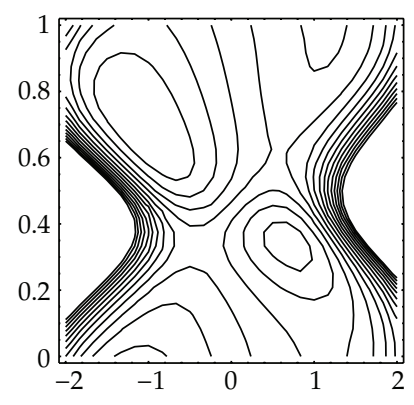

(c)

Figure 18: Streamlines for three different values of $b$ : (a) $b=0.1$, (b) $b=0.3$, (c) $b=0.5$. The other parameters chosen are $\Theta=4, d=1, M=1.2, a=0.4, m=1.5, \beta=-2, k=0.5, \phi=\pi / 4, \mathrm{Gr}=2$.

(i) The velocity increases by increasing each of $m, k, \mathrm{Gr}, \phi, a$, and $\beta$ and decreases by increasing $d$ and $b$.

(ii) The pressure gradient decreases with the increase of $m, k, \phi$, and d while it increases by increasing $\mathrm{Gr}, a, b$, and $\beta$.

(iii) Increasing each of $m, k, \beta$, and Gr led to decreasing the shear stress at the right wall but it increases by increasing $d$.

(iv) The shear stress at the left wall increases by increasing each of $m, k, \beta$, and Gr and decreases by increasing $d$.

(v) An increase in Gr and $\theta$ led to an increase in the pressure rise $\Delta p_{\lambda}$.

(vi) The friction force at the right wall decreases by increasing Gr and $\beta$.

(vii) The friction force at the left wall increases by increasing Gr and $\beta$.

(viii) Increasing $m$ and $k$ increases the friction forces in some regions and decreases the friction forces in some other regions.

(ix) The heat transfer coefficient decreases by increasing $d$ and $\phi$ and increases by increasing $\beta$. 


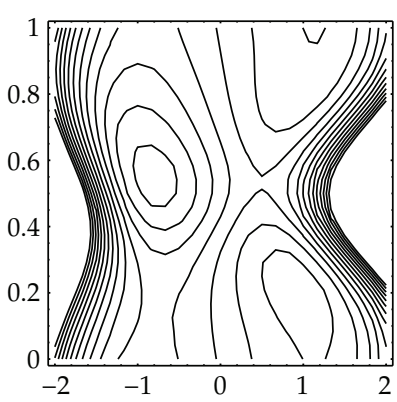

(a)

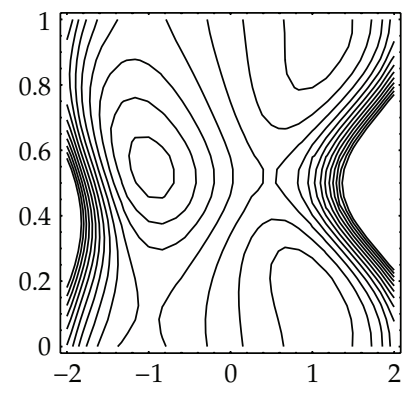

(b)

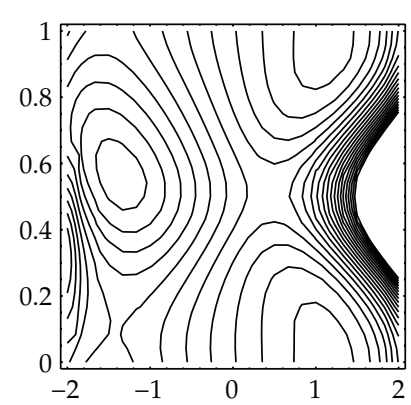

(c)

Figure 19: Streamlines for three different values of $d$ : (a) $d=1$, (b) $d=1.2$, (c) $d=1.6$. The other parameters chosen are $\Theta=4, m=1.5, M=1.2, a=0.4, b=0.2, \beta=-2, k=0.5, \phi=\pi / 4, \mathrm{Gr}=2$.

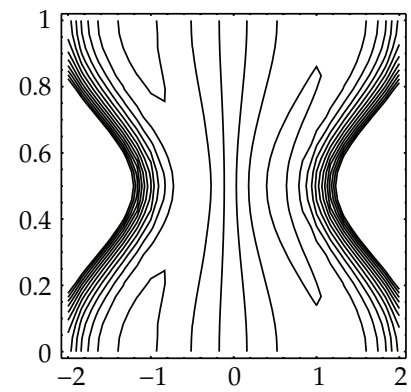

(a)

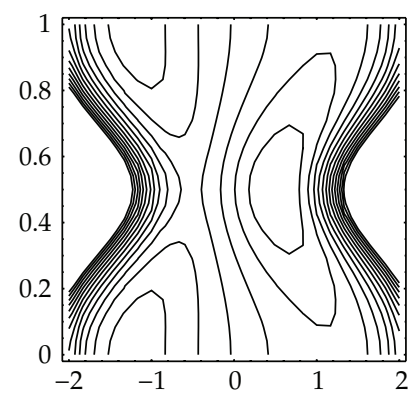

(d)

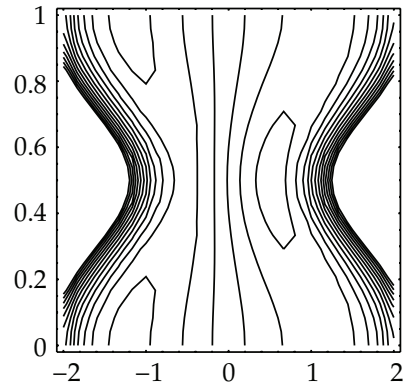

(b)

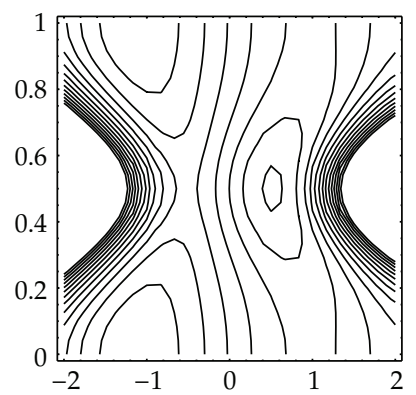

(e)

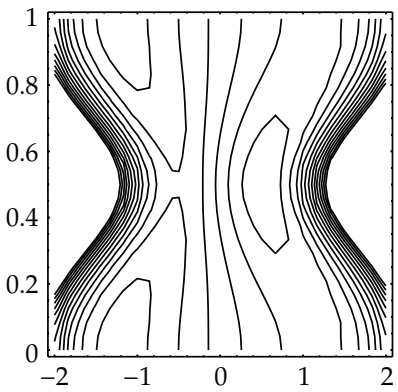

(c)

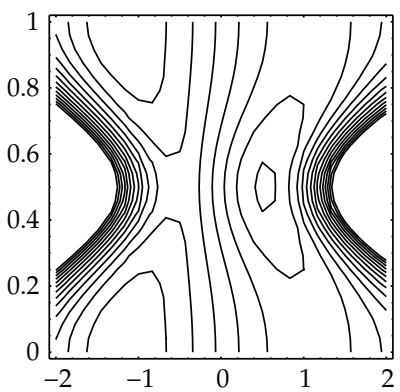

(f)

Figure 20: Streamlines for symmetric channel $(a=b=0.4, d=1, \phi=0)$. The other parameters chosen are (a) $\Theta=4, \beta=-2, m=0.5, k=0.5, M=1.5, \mathrm{Gr}=2$; (b) $\Theta=4, \beta=-2, m=0.5, k=0.5, M=1.5, \mathrm{Gr}=5$; (c) $\Theta=4, \beta=-2, m=1.5, k=0.5, M=1.5, \mathrm{Gr}=5$; (d) $\Theta=4, \beta=-2, m=1.5, k=0.8, M=1.5, \mathrm{Gr}=5$; (e) $\Theta=4, \beta=2, m=1.5, k=0.8, M=1.5, \mathrm{Gr}=5$; (f) $\Theta=5, \beta=2, m=1.5, k=0.8, M=1.5, \mathrm{Gr}=5$.

(x) The size of trapped bolus increases by increasing $m, \Theta, G r, k, a$, and $d$ and decreases by increasing $\beta$ and $b$.

(xi) In the case of symmetric channel the size of trapped bolus increases by increasing each of $m, k$, and $\Theta$ and decreases by increasing each of $\mathrm{Gr}$ and $\beta$. 


\section{Acknowledgment}

This project was supported by King Saud University, Deanship of Scientific Research, College of Science, Research Center. The authors would like to express their thanks to both the referee and the editor for their kind and helpful comments.

\section{References}

[1] T. W. Latham, Motion in a Peristaltic Pump, M.S. thesis, MIT-Press, Cambridge, Mass, USA, 1966.

[2] Y. C. Fung and C. S. Yih, "Peristaltic transport," Journal of Applied Mechanics, vol. 35, no. 4, pp. 669-675, 1968.

[3] A. H. Shapiro, M. Y. Jaffrin, and S. L. Weinberg, "Peristaltic pumping with long wavelengths at low Reynolds number," Journal of Fluid Mechanics, vol. 37, no. 4, pp. 799-825, 1969.

[4] M. Y. Jaffrin and A. H. Shapiro, "Peristaltic pumping," Annual Review of Fluid Mechanics, vol. 3, no. 1, pp. 13-36, 1971.

[5] L. M. Srivastava and V. P. Srivastava, "Peristaltic transport of blood: casson model. II," Journal of Biomechanics, vol. 17, no. 11, pp. 821-829, 1984.

[6] H. L. Agrawal and B. Anwaruddin, "Peristaltic flow of blood a branch," Ranchi University Mathematical Journal, vol. 15, pp. 111-118, 1984.

[7] E. F. El-Shehawey and S. Z. A. Husseny, "Peristaltic transport of a magneto-fluid with porous boundaries," Applied Mathematics and Computation, vol. 129, no. 2-3, pp. 421-440, 2002.

[8] A. M. Siddiqui, T. Hayat, and M. Khan, "Magnetic fluid model induced by peristaltic waves," Journal of the Physical Society of Japan, vol. 73, no. 8, pp. 2142-2147, 2004.

[9] A. E. H. A. El Naby, A. E. M. El Misery, and M. F. Abd El Kareem, "Effects of a magnetic field on trapping through peristaltic motion for generalized Newtonian fluid in channel," Physica A, vol. 367, pp. 79-92, 2006.

[10] T. Hayat, M. Javed, and N. Ali, "MHD peristaltic transport of a Jeffery fluid in a channel with compliant walls and porous space," Transport in Porous Media, vol. 74, no. 3, pp. 259-274, 2008.

[11] K. S. Mekheimer, "Non-linear peristaltic transport of magnetohydrodynamic flow in an inclined planar channel," Arabian Journal for Science and Engineering A, vol. 28, no. 2, pp. 183-201, 2003.

[12] K. S. Mekheimer, "Peristaltic flow of blood under effect of a magnetic field in a non-uniform channels," Applied Mathematics and Computation, vol. 153, no. 3, pp. 763-777, 2004.

[13] A. E. H. A. El Naby, A. E. M. El Misery, and M. F. Abd El Kareem, "Effects of a magnetic field on trapping through peristaltic motion for generalized Newtonian fluid in channel," Physica A, vol. 367, pp. 79-92, 2006.

[14] T. Hayat, M. Javed, and N. Ali, "MHD peristaltic transport of a Jeffery fluid in a channel with compliant walls and porous space," Transport in Porous Media, vol. 74, no. 3, pp. 259-274, 2008.

[15] M. Mishra and A. Ramachandra Rao, "Peristaltic transport of a Newtonian fluid in an asymmetric channel," Zeitschrift fur Angewandte Mathematik und Physik, vol. 54, no. 3, pp. 532-550, 2003.

[16] M. H. Haroun, "Non-linear peristaltic transport flow of a fourth grade fluid in an inclined asymmetric channel," Computational Materials Science, vol. 39, pp. 324-333, 2007.

[17] K. Vajravelu, S. Sreenadh, and P. Lakshminarayana, "The influence of heat transfer on peristaltic transport of a Jeffrey fluid in a vertical porous stratum," Communications in Nonlinear Science and Numerical Simulation, vol. 16, no. 8, pp. 3107-3125, 2011.

[18] E. F. El Shehawey and S. Z. A. Husseny, "Effects of porous boundaries on peristaltic transport through a porous medium," Acta Mechanica, vol. 143, no. 3, pp. 165-177, 2000.

[19] E. F. El Shehawey, N. T. Eldabe, E. M. Elghazy, and A. Ebaid, "Peristaltic transport in an asymmetric channel through a porous medium," Applied Mathematics and Computation, vol. 182, no. 1, pp. 140-150, 2006.

[20] K. Vajravelu, G. Radhakrishnamacharya, and V. Radhakrishnamurty, "Peristaltic flow and heat transfer in a vertical porous annulus, with long wave approximation," International Journal of NonLinear Mechanics, vol. 42, no. 5, pp. 754-759, 2007.

[21] S. Srinivas and M. Kothandapani, "The influence of heat and mass transfer on MHD peristaltic flow through a porous space with compliant walls," Applied Mathematics and Computation, vol. 213, no. 1, pp. 197-208, 2009. 
[22] S. Srinivas and R. Gayathri, "Peristaltic transport of a Newtonian fluid in a vertical asymmetric channel with heat transfer and porous medium," Applied Mathematics and Computation, vol. 215, no. 1, pp. 185-196, 2009.

[23] H. A. Attia, "Unsteady hartmann flow with heat transfer of a viscoelastic fluid considering the hall effect," Canadian Journal of Physics, vol. 82, no. 2, pp. 127-139, 2004.

[24] S. Asghar, M. R. Mohyuddin, and T. Hayat, "Effects of Hall current and heat transfer on flow due to a pull of eccentric rotating disks," International Journal of Heat and Mass Transfer, vol. 48, no. 3-4, pp. 599-607, 2005.

[25] T. Hayat, N. Ali, and S. Asghar, "Hall effects on peristaltic flow of a Maxwell fluid in a porous medium," Physics Letters A, vol. 363, no. 5-6, pp. 397-403, 2007.

[26] E. M. Abo-Eldahab, E. I. Barakat, and K. I. Nowar, "Hall currents and ion-slip effects on the MHD peristaltic transport," International Journal of Applied Mathematics and Physics, vol. 2, pp. 113-123, 2010.

[27] E. M. Abo-Eldahab, E. I. Barakat, and K. I. Nowar, "effects of Hall and ion-slip currents on peristaltic transport of a couple stress fluid," International Journal of Applied Mathematics and Physics, vol. 2, no. 2, pp. 145-157, 2010.

[28] N. S. Akbar and S. Nadeem, "Influence of heat transfer on peristaltic transport of a JohnsonSegalman fluid in an inclined asymmetric channel," Communications in Nonlinear Science and Numerical Simulation, vol. 15, no. 10, pp. 2860-2877, 2010.

[29] N. Ali, M. Sajid, T. Javed, and Z. Abbas, "Heat transfer analysis of peristaltic flow in a curved channel," International Journal of Heat and Mass Transfer, vol. 53, no. 15-16, pp. 3319-3325, 2010.

[30] K. S. Mekheimer, N. Saleem, T. Hayat, and A. A. Hendi, "Simultaneous effects of induced magnetic field and heat and 16 mass transfer on the peristaltic motion of second-order fluid in a channel," International Journal for Numerical Methods in Fluids. In press.

[31] K. S. Mekheimer and A. N. Abdel-Wahab, "Net annulus flow of a compressible viscous liquid with peristalsis," Journal of Aerospace Engineering. In press.

[32] Y. Abd El-Maboud and K. S. Mekheimer, "Non-linear peristaltic transport of a second-order fluid through a porous medium," Applied Mathematical Modelling, vol. 35, no. 6, pp. 2695-2710, 2010.

[33] K. S. Mekheimer, S. A. Husseny, and Y. Abd El-Maboud, "Effects of heat transfer and space porosity on peristaltic flow in a vertical asymmetric channel," Numerical Methods of Partial Differential Equations Journal, vol. 26, no. 4, pp. 747-770, 2010.

[34] Z. M. Gharsseldien, K. S. Mekheimer, and A. S. Awad, "The influence of slippage on trapping and reflux limits with peristalsis through an asymmetric channel," Applied Bionics and Biomechanics, vol. 7, no. 2, pp. 95-108, 2010.

[35] S. Srinivas and R. Muthuraj, "Effects of chemical reaction and space porosity on MHD mixed convective flow in a vertical asymmetric channel with peristalsis," Mathematical and Computer Modelling, vol. 54, no. 5-6, pp. 1213-1227, 2011. 


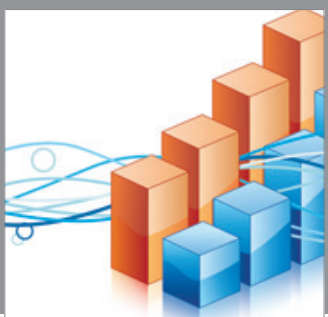

Advances in

Operations Research

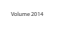

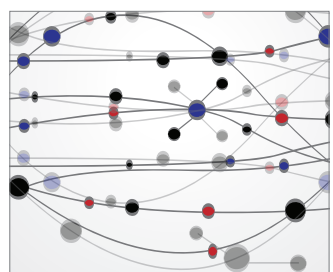

\section{The Scientific} World Journal
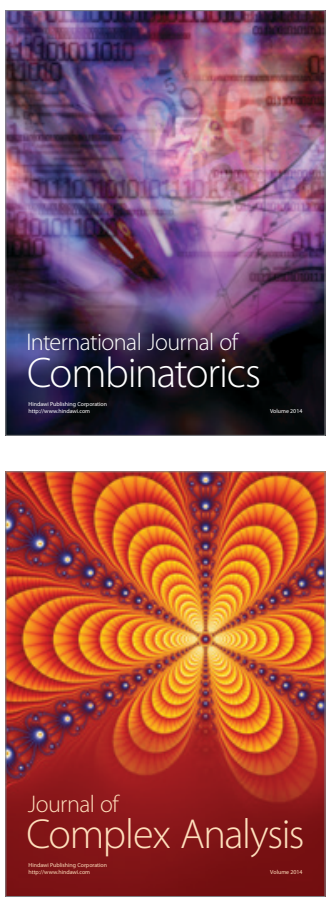

International Journal of

Mathematics and

Mathematical

Sciences
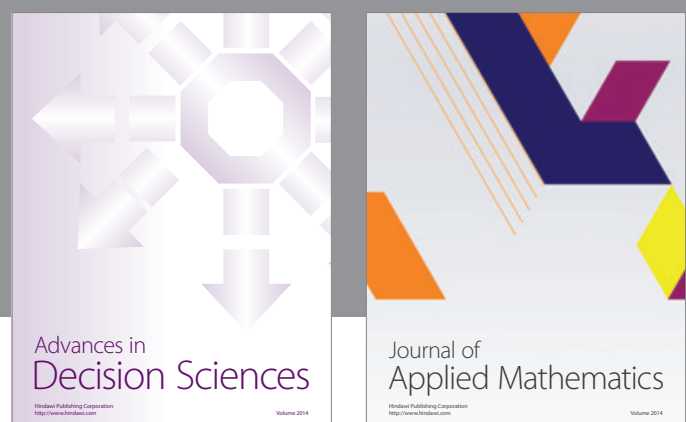

Journal of

Applied Mathematics
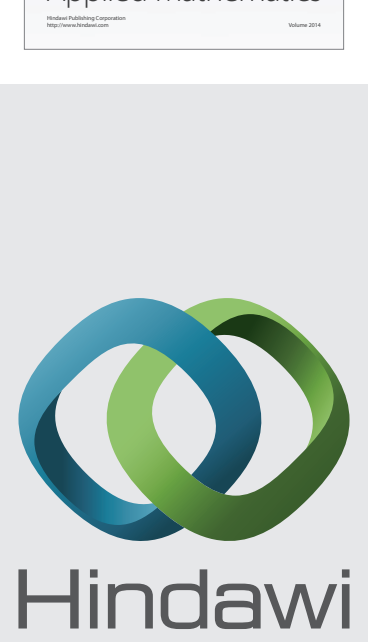

Submit your manuscripts at http://www.hindawi.com
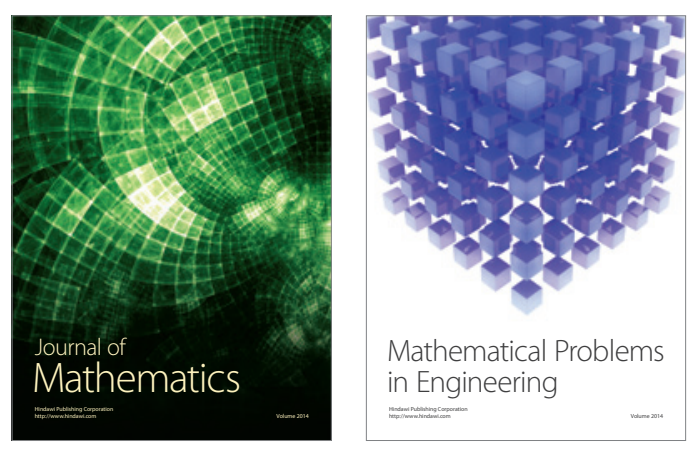

Mathematical Problems in Engineering
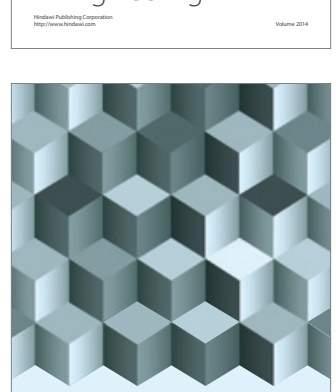

Journal of

Function Spaces
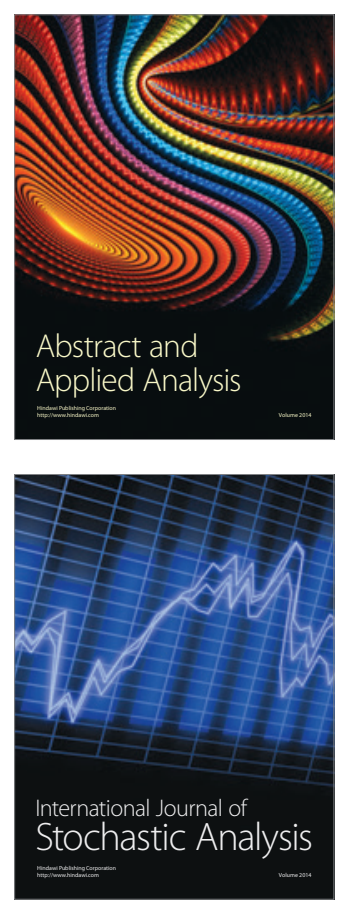

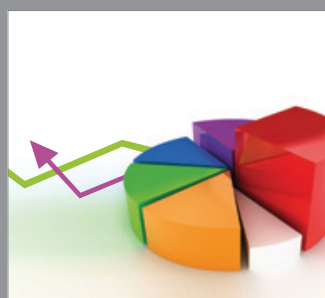

ournal of

Probability and Statistics

Promensencen
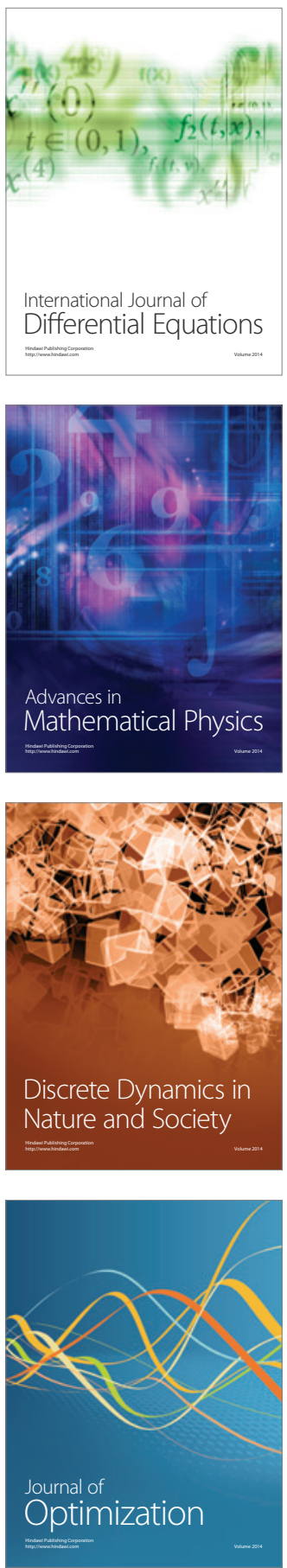